\title{
Equation-of-motion coupled-cluster method with double electron-attaching operators: Theory, implementation, and benchmarks
}

\author{
Sahil Gulania ${ }^{a}$, Eirik F. Kjønstad ${ }^{b}$, John F. Stanton ${ }^{c}$, Henrik Koch ${ }^{b, d}$, Anna I. Krylov ${ }^{a}$ \\ ${ }^{a}$ Department of Chemistry, University of Southern California, Los Angeles, California 90089, USA \\ ${ }^{b}$ Department of Chemistry, Norwegian University of Science and Technology, 7491 Trondheim, Norway \\ ${ }^{c}$ Quantum Theory Project, Departments of Chemistry and Physics, University of Florida, Gainesville, Florida 32611, USA \\ $d$ Scuola Normale Superiore, Piazza dei Cavaleri 7, 56126 Pisa, Italy
}

(Dated: 24 December 2020)

We report a production-level implementation of equation-of-motion coupled-cluster method with double electronattaching EOM operators of $2 p$ and $3 p 1 h$ types, EOM-DEA-CCSD. This ansatz, suitable for treating electronic structure patterns that can be described as two-electrons-in-many orbitals, represents a useful addition to EOM-CC family of methods. We analyze the performance of EOM-DEA-CCSD for energy differences and molecular properties. By considering reduced quantities, such as state and transition one-particle density matrices, we can compare EOM-DEACCSD wave functions with wave functions computed by other EOM-CCSD methods. The benchmarks illustrate that EOM-DEA-CCSD is capable of treating diradicals, bond-breaking, and some types of conical intersections.

\section{INTRODUCTION}

The robust black-box treatment of open-shell and electronically excited species remains elusive, despite the progress in our understanding of electron correlation and advances in algorithms and computer hardware. The essential challenge in designing a general strategy is a great diversity of open-shell patterns, the same trait that is responsible for chemical versatility of transition metals, multiple roles of reaction intermediates, and vast variety of photoinduced reactions. The great variety of open-shell patterns can be adequately captured, in general, by only the exact treatment, full configuration interaction (FCI). More compact formulations rely on specific approximations, which are grounded in concrete physics and, therefore, have a limited scope of applicability. Nevertheless, quantum chemistry has developed several uniform and welldefined approaches for treating broad classes of electronic structure.

The coupled-cluster (CC) hierarchy of approximations ${ }^{1,2}$ provides the best set of tools for ground states of molecules with moderate non-dynamical correlation, such as closedshell molecules with large spectral gaps or simple open-shell species (i.e., such as high-spin states with the maximal spin projection). If the wave function is well described by a single Slater determinant, then CC methods account for dynamical correlation with an impressive accuracy achieved already at low-level many-body treatments. ${ }^{3,4}$

Equation-of-motion coupled-cluster (EOM-CC) theory ${ }^{5-15}$ provides an extension of CC theory to open-shell species and electronically excited states. EOM-CC theory is not a single method; rather, it is a general framework. In the Fockspace formalism, it provides a strategy for describing different classes of electronic structure in an efficient and robust manner. Different variants of EOM-CC provide access to different types of target electronic states, such as electronically excited, electron attached, or ionized states. It is important to note that the description of the target states relates to the electronic structure pattern and not to a particular phenomenon or experiment. For example, in the EOM terminology, electronattached states refer to the states that can be described as the result of adding an electron to a closed-shell reference and not to the states produced by actual attachment of an electron to a molecule.

In this contribution, we focus on a variant of EOM-CC targeting electronic structure that can be described as 2electrons-in-many orbitals pattern, as observed in diradicals and molecules with small HOMO-LUMO gaps. This approach, which was introduced ${ }^{16}$ by Nooijen and Bartlett for similarity transformed EOM in 1997, was later further developed $^{17}$ and used in several illustrative calculations. ${ }^{18-21}$ However, its full potential has not been appreciated by the computational chemistry community. Here we report an efficient implementation, including energies and properties, and illustrate the capabilities of this method by a broad set of examples. The examples highlight the ability of EOM-CC to treat a variety of situations, often described as 'multi-reference', in an efficient and robust single-reference framework $^{12,14,22}$. In particular, EOM-DEA is a useful tool for treating diradicals, doubly excited states, bond-breaking, and some types of conical intersections.

\section{THEORY}

The EOM-CC wave function is expressed as

$$
|\Psi\rangle=R e^{T}\left|\Phi_{0}\right\rangle
$$

where the linear operator $R$ acts on the reference CC wave function, $e^{T}\left|\Phi_{0}\right\rangle$. The operator $T$ is an excitation operator satisfying the $\mathrm{CC}$ equations for the reference state,

$$
\left\langle\Phi_{\mu}|\bar{H}| \Phi_{0}\right\rangle=0
$$

where $\bar{H}=e^{-T} H e^{T}$ and $\Phi_{\mu}$ are the $\mu$-tuply excited determinants with respect to the reference determinant $\Phi_{0}$. In EOMCCSD, the CC operator is truncated as ${ }^{3}$

$$
T \approx T_{1}+T_{2}
$$


where $T_{1}$ and $T_{2}$ are spin- and particle-conserving single and double excitation operators of 1-hole-1-particle $(1 h 1 p)$ and 2holes-2-particles $(2 h 2 p)$ types:

$$
T_{1}=\sum_{i a} t_{i}^{a} a^{\dagger} i, \quad T_{2}=\frac{1}{4} \sum_{i j a b} t_{i j}^{a b} a^{\dagger} b^{\dagger} j i
$$

and the truncation of $R$ is done in a consistent manner.

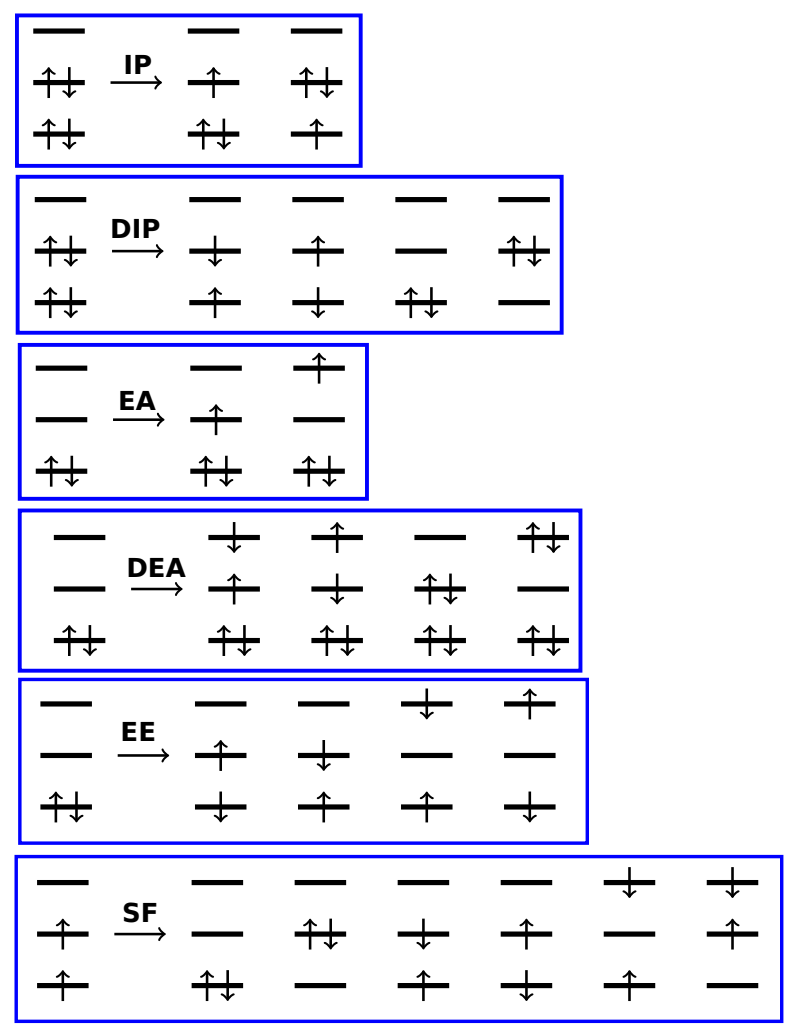

FIG. 1. Different types of target states can be accessed by different combination of the reference state and EOM operators. Reproduced with permission from Ref. 23.

Different variants ${ }^{12-14}$ of EOM-CC are defined by different choices of the reference state and the type of EOM operators $R$, as illustrated in Fig. 1. The focus of this work is on EOMDEA. ${ }^{17,21,24}$ In this method, the operators $R_{1}$ and $R_{2}$ are of $2 p$ and $1 h 3 p$ types:

$$
\begin{aligned}
& R_{1}=\frac{1}{2} \sum_{a b} r^{a b} a^{\dagger} b^{\dagger} \\
& R_{2}=\frac{1}{6} \sum_{i a b c} r_{i}^{a b c} a^{\dagger} b^{\dagger} c^{\dagger} i .
\end{aligned}
$$

The EOM amplitudes and the corresponding energies are found by diagonalizing the matrix of the similarity transformed Hamiltonian, $\bar{H}$, in the basis of determinants generated by the EOM operators $R$ acting on the reference $\Phi_{0}$. Since $\bar{H}$ is a non-Hermitian operator, its left and right eigenstates are not Hermitian conjugates but form a biorthonormal set:

$$
\begin{gathered}
\bar{H} R\left|\Phi_{0}\right\rangle=E R\left|\Phi_{0}\right\rangle \\
\left\langle\Phi_{0}\right| L \bar{H}=\left\langle\Phi_{0}\right| L E
\end{gathered}
$$

$$
\left\langle\Phi_{0}\left|L^{M} R^{N}\right| \Phi_{0}\right\rangle=\delta_{M N}
$$

where $M$ and $N$ denote the $M$ th and $N$ th EOM states and

$$
L=L_{1}+L_{2}=\frac{1}{2} \sum_{a b} l^{a b} b a+\frac{1}{6} \sum_{i a b c} l_{i}^{a b c} i^{\dagger} c b a .
$$

Here and below $p^{\dagger}$ and $q$ are electron creation and annihilation operators corresponding to orbitals $\phi_{p}$ and $\phi_{q}$ (following the standard convention, indices $i, j, k \ldots$ denote orbitals occupied in $\Phi_{0}, a, b, c, \ldots$ denote virtual orbitals, and $p, q, r, \ldots$ denote orbitals from both subspaces). For energy calculations right eigenstates are sufficient but for property calculations both left and right eigenstates need to be computed.

The left and right amplitudes are found by diagonalizing the corresponding matrix representation of $\bar{H}$. Hence, an EOMCCSD calculation entails the diagonalization of the effective Hamiltonian $\bar{H}$ in the basis of the reference and singly and doubly excited determinants, which can be written in the matrix form as

$$
\left(\begin{array}{cc}
\bar{H}_{S S}-E_{c c} & \bar{H}_{S D} \\
\bar{H}_{D S} & \bar{H}_{D D}-E_{c c}
\end{array}\right)\left(\begin{array}{l}
R_{1} \\
R_{2}
\end{array}\right)=\omega\left(\begin{array}{l}
R_{1} \\
R_{2}
\end{array}\right)
$$

and

$$
\left(\begin{array}{ll}
L_{1} & L_{2}
\end{array}\right)\left(\begin{array}{cc}
\bar{H}_{S S}-E_{c c} & \bar{H}_{S D} \\
\bar{H}_{D S} & \bar{H}_{D D}-E_{c c}
\end{array}\right)=\omega\left(\begin{array}{ll}
L_{1} & L_{2},
\end{array}\right)
$$

where $\omega$ is the energy difference with respect to the reference coupled-cluster state. Note that the structure of the effective Hamiltonian ensures size-intensivity, but not sizeextensivity. ${ }^{25}$ In practice, Eqns. (9) and (10) are solved iteratively, using a generalization of Davidson's diagonalization procedure. This procedure requires calculation of the Hamiltonian acting on trial vectors; the relevant expressions are given in Appendix A.

The scaling of the EOM part in the standard EOM-DEACCSD implementation is $N^{6}\left(O^{1} V^{5}\right)$, to be compared with $O^{2} V^{4}$ in EOM-EE/SF-CCSD, $O^{3} V^{2}$ in EOM-IP-CCSD, $O^{4} V^{2}$ in EOM-DIP-CCSD, and $O^{1} V^{4}$ in EOM-EA-CCSD. The EOM-DEA-CCSD scaling is determined by the contraction between the transformed two-electron integral and $R_{2}$ or $L_{2}$ vectors $\left(\sum_{d e} I_{a b d e}^{5} r_{i}^{c d e}\right.$ or $\left.\sum_{d e} l_{i}^{a d e} I_{d e b c}^{5}\right)$ entering the calculation of the doubles-doubles block (see Table XXII).

To analyze EOM-CCSD wave functions, we employ reduced quantities such as state and transition one-particle density matrices. These objects are related to concrete physical observables ${ }^{26}$ and, therefore, provide insight into underlying electronic structure and facilitate comparison between different methods ${ }^{27}$. They also provide a way to derive a molecular orbital picture ${ }^{26}$ of many-body wave functions by using 
concepts such as natural orbitals (NOs) and natural transition orbitals (NTOs).

The $\mathrm{NOs}^{28}$ provide a compact one-particle representation of many-electron wave functions. They are defined as eigenstates of one-particle (state) density matrices (1PDMs). In second quantization, the $1 \mathrm{PDM}\left(\gamma^{I}\right)$ for the $\Psi_{I}$ state is defined as

$$
\gamma_{p q}^{I}=\left\langle\Psi_{I}\left|p^{\dagger} q\right| \Psi_{I}\right\rangle
$$

The eigenvalues of 1PDM are called occupation numbers $\left(n_{i}\right)$; they range from 0 to 1 and add up to the total number of the electrons. 1PDMs contain all information necessary to compute the expectation value of any one-particle operator $\hat{O}_{1}$ (dipole moment $\mu$, size of wave function $\mathrm{R}^{2}$, etc):

$$
\left\langle\Psi_{I}\left|\hat{O}_{1}\right| \Psi_{I}\right\rangle=\sum_{p q} \gamma_{p q}^{I}\left\langle\phi_{p}\left|\hat{O}_{1}\right| \phi_{q}\right\rangle .
$$

1PDMs can be also used to extract quantities that are not related to physical observables, but provide insight into electronic structure, such as the number of effectively unpaired electrons. ${ }^{29-31}$ Here we use $n_{u, n l}$ index, proposed by HeadGordon, ${ }^{30}$

$$
n_{u, n l}=\sum_{i} \bar{n}_{i}^{2}\left(2-\bar{n}_{i}\right)^{2}
$$

where $\bar{n}_{i}$ are spin-averaged occupation numbers.

Similarly, one can define the one-particle transition density matrix $^{27,32-36}$ (1PTDM) between two many-electron wave functions $\Psi_{I}$ and $\Psi_{J}$ :

$$
\gamma_{p q}^{I J}=\left\langle\Psi_{J}\left|p^{\dagger} q\right| \Psi_{I}\right\rangle
$$

The norm of $\gamma^{I J}$ provides a measure on one-electron character of the transition (e.g., $\|\gamma\|=1$ for transitions between $\Phi_{0}$ and a CIS excited state). The 1PTDM provides a compact representation of the difference between the two states in terms of hole-particle excitations. Singular value decomposition of $\gamma^{I J}$ provides a set of left and right eigenvectors

$$
\gamma^{I J}=U \Sigma V^{T}
$$

which define hole $\left(\psi_{K}^{h}\right)$ and particle $\left(\psi_{K}^{e}\right)$ orbitals corresponding to singular value $\sigma_{K}$

$$
\begin{aligned}
\psi_{K}^{h} & =\sum_{q} U_{q K} \phi_{q}, \\
\psi_{K}^{e} & =\sum_{q} V_{q K} \phi_{q} .
\end{aligned}
$$

Such pairs of hole and particle orbitals are called NTOs. Usually, only a small number of singular values are significant. To quantify the collectivity of excitation (i.e., how many NTO pairs are needed to describe the transition), we use the participation ratio defined as

$$
\mathrm{PR}_{N T O}=\frac{\left(\sum_{i} \sigma_{i}\right)^{2}}{\sum_{i} \sigma_{i}^{2}}=\frac{\|\gamma\|^{2}}{\sum_{i} \sigma_{i}^{2}} .
$$

The participation ratio is closely related to the number of entangled states, an alternative metric ${ }^{37}$ also defined on the basis of the 1PTDMs:

$$
Z_{H E}=2^{S_{H E}}=1 / \prod_{i} \lambda_{i}^{\lambda_{i}}
$$

where $\lambda_{i}=\sigma_{i}^{2} /\|\gamma\|^{2}$ are renormalized squares of the singular values of 1PTDM and $S_{H E}$ is the hole-electron entanglement entropy:

$$
S_{H E}=-\sum_{i} \lambda_{i} \log _{2} \lambda_{i} .
$$

To properly account for spin entanglement in spin-adapted basis, we use the extensivity of entropy, i.e., the total $S_{H E}$ is the sum of the $\alpha \alpha$ and $\beta \beta$ parts of the transition. Therefore, when using restricted Hartree-Fock references, one needs to add $S_{H E}$ from the $\alpha \alpha$ and $\beta \beta$ spin-sectors to obtain total $S_{H E}$.

The NTOs and the respective exciton descriptors provide convenient tools to analyze the transitions between states $^{26,27,38}$, as long as they have predominantly one-electron character (i.e., when $\|\gamma\| \sim 1$ ).

\section{RESULTS AND DISCUSSION}

To illustrate the scope of applicability of EOM-DEACCSD, we consider the following examples:

- Low-lying states in molecules with diradical character ${ }^{11,39-41}: \mathrm{CH}_{2}$, benzynes, cyclobutadiene. For these systems, we compare EOM-DEA with EOM-SF. We consider both energy differences and properties. For the diradical manifold (such as low-lying singlets and triplets), we anticipate similar performance; however, EOM-DEA can also access higher excited states, as was illustrated in recent studies of Rydberg diradicals ${ }^{42,43}$.

- Ground and excited state of ozone. This example illustrates both the advantages and the limitations of the EOM-DEA ansatz. Depending on the chosen reference state chosen, different target manifolds are accessible by EOM-DEA.

- Ground and excited states of ethylene at equilibrium geometry and along torsional coordinate. This example illustrates $(\pi)$ bond-breaking and the ability of EOMDEA to treat doubly excited states.

- Excited states of butadiene. This examples illustrates the extent of doubly excited character in the lowest dark state in polyenes.

- To assess the ability of EOM-DEA to treat conical intersections, we consider a well studied retinal example ${ }^{44-46}$ and $\mathrm{HeH}_{2}$.

- Excited states in small molecules (water and ammonia), where we compare EOM-DEA with EOM-EE and other methods, including FCI. 
Similarly to the ozone case, these examples illustrate that states that are dominated by excitations from HOMO are described well by EOM-DEA, but states derived by excitations from lower orbitals are not.

Section III A provides computational details for each example.

\section{A. Computational details}

All EOM-CCSD calculations were performed using the QChem electronic structure program ${ }^{47,48}$. The reported symmetry labels of electronic states and MOs correspond to Mulliken's convention ${ }^{49}$, which differs from the standard molecular orientation used in Q-Chem (hence, some state labels had to be changed). The EOM-CCSDT calculations were performed with $\mathrm{CFOUR}^{50}$ and the EOM-CC3 calculations with eT. ${ }^{51,52}$ For $\mathrm{HeH}_{2}$, EOM-EE-CCSD calculations were performed with eT and FCI calculations with DALTON. ${ }^{53}$ In EOM-DEA-CCSD calculations, we use default $N$-2 electron references. To investigate effects of orbital relaxation, we also carried out calculations with orbitals computed for $N$-electron states. In cases when different orbitals were used, the charge of the reference state is indicated as follows: 'EOM-DEA$\mathrm{CCSD} /+\mathrm{X}$ ', where ' $+\mathrm{X}$ ' denotes the charge of the HartreeFock determinant defining the orbitals used in the EOM-CC calculations.

Methylene calculations were performed using the equilibrium geometries and TZ2P basis set from Ref. 54. Pure angular momentum polarization functions were employed. In CCSD and EOM calculations, all orbitals were active. Note that the reference FCI energies ${ }^{54}$ were computed with one frozen core and one frozen virtual orbital. Cyclobutadiene calculations were performed using the same equilibrium geometries as in Ref. 11. All calculations were performed with the cc-pVTZ basis set. Pure angular momentum polarization functions were employed; all orbitals were active. Calculations of benzynes were performed using the same equilibrium geometries as in Ref. 55 and with the cc-pVTZ basis set. Pure angular momentum polarization functions were employed; all orbitals were active.

Butadiene calculations were performed using the geometries from Ref. 56. Pure angular momentum polarization functions were employed; core orbitals were frozen. Ethylene calculations were performed using the geometries from Ref. 57. Cartesian polarization functions were employed; core electrons were frozen. Ozone calculations were performed using the geometries from Ref. 58. Pure angular momentum functions were employed. Core electrons were frozen.

Water and ammonia calculations were performed using the geometries from Ref. 59. Pure angular momentum functions were employed; core electrons were frozen. Retinal chromophore calculations were performed using the geometries from Ref. 44. Cartesian polarization functions were employed; all electrons were active. $\mathrm{HeH}_{2}$ calculations are performed using pure angular momentum functions; all electrons were active.
Computation of $Z_{H E}$ requires additional clarification. When using restricted Hartree-Fock references, the $Q$ Chem/libwfa output of renormalized entanglement entropy $\left(S_{H E}\right)$ for the transition should be multiplied by 2 . When using unrestricted Hartree-Fock references, $S_{H E}$ is taken directly from the Q-Chem/libwfa output. Then $Z_{H E}$ is computed as $2^{S_{H E}}$

\section{B. $\mathrm{CH}_{2}$}

Methylene is an example of a simple diradical with a triplet ground state. Its low-lying electronic states are derived by distributing two electrons over two frontier orbitals $\left(p_{z}\right.$ and $s p^{2}$-hybridized orbitals of carbon). Table I shows the total energy for the $\tilde{X}^{3} \mathrm{~B}_{1}$ state (high-spin) and adiabatic excitation energies for the $\tilde{a}^{1} \mathrm{~A}_{1}, \tilde{b}^{1} \mathrm{~B}_{1}$, and $\tilde{b}^{1} \mathrm{~B}_{1}$ states computed with EOM-DEA-CCSD and several other methods.

TABLE I. Total energies (hartree) for the ground $\tilde{X}^{3} \mathrm{~B}_{1}$ state of $\mathrm{CH}_{2}$ and adiabatic excitation energies $(\mathrm{eV})$ for the three lowest singlet states $^{a}$. ZPE not included; TZ2P basis set.

\begin{tabular}{|c|c|c|c|}
\hline Method & $\tilde{X}^{3} \mathrm{~B}_{1}$ & $\tilde{a}^{1} \mathrm{~A}_{1} \quad \tilde{b}^{1} \mathrm{~B}_{1}$ & $\tilde{c}^{1} \mathrm{~A}_{1}$ \\
\hline $\mathrm{HF}^{b}$ & -38.937956 & 1.236 & 2.772 \\
\hline $\mathrm{CCSD}^{b}$ & -39.080919 & 0.545 & 2.054 \\
\hline $\operatorname{CCSD}(\mathrm{T})^{b}$ & -39.083856 & 0.505 & 1.907 \\
\hline $\mathrm{EOM}^{-\mathrm{EE}-\mathrm{CCSD}}{ }^{b}$ & -39.08066 & 0.5381 .566 & 3.843 \\
\hline $\mathrm{SF}-\mathrm{CIS}{ }^{b}$ & -38.93254 & 0.8831 .875 & 3.599 \\
\hline SF-CIS(D) $)^{b}$ & -39.05586 & 0.6131 .646 & 2.953 \\
\hline $\mathrm{EOM}-\mathrm{SF}-\mathrm{OD}^{b}$ & -39.08045 & 0.5141 .564 & 2.715 \\
\hline EOM-SF-CCSD/UHF ${ }^{c}$ & -39.080453 & 0.5171 .565 & 2.718 \\
\hline EOM-SF-CCSD(fT)/UHF ${ }^{c}$ & -39.08184 & 0.5001 .552 & 2.688 \\
\hline $\mathrm{EOM}-\mathrm{SF}-\mathrm{CCSD}(\mathrm{dT}) / \mathrm{UHF}^{c}$ & -39.08217 & 0.4961 .548 & 2.678 \\
\hline $\mathrm{EOM}-\mathrm{DEA}-\mathrm{CCSD}^{d}$ & -39.069681 & 0.4811 .461 & 2.518 \\
\hline $\mathrm{FCI}^{e}$ & -39.066738 & 0.4831 .542 & 2.674 \\
\hline
\end{tabular}

${ }^{a} \mathrm{FCI} / \mathrm{TZ2P}$ optimized geometries. All electrons were active in CC/EOM-CC calculations. ${ }^{b}$ Computed using closed-shell singlet reference; from Ref. 39. ${ }^{c}$ From Ref. 41.

$d$ This work. ${ }^{e}$ From Ref. 54; one frozen core and one frozen virtual orbital.

As expected, Hartree-Fock calculations overestimate the singlet-triplet gap because of an unbalanced description of the singlet state (which needs more correlation because of the diradical character) and the triplet state (which is well described by the single determinant). However, the diradical character in the lowest singlet state is modest, so that $\triangle$ CCSD calculation yields a reasonable gap, which is further improved by including triples corrections. We note that the EOM-EECCSD calculation (performed using a closed-shell reference) yields a better value of the gap than CCSD, because of a more balanced description of the two states. Another advantage of EOM-CC ansatz is that it yields not just the lowest triplet, but also two other singlet states. The energy of the openshell singlet, $\tilde{b}^{1} \mathrm{~B}_{1}$ is reproduced well by EOM-EE-CCSD, 
TABLE II. One-electron properties for the lowest electronic states of $\mathrm{CH}_{2}$ computed using EOM-SF-CCSD and EOM-DEA-CCSD wave functions; aug-cc-pVTZ basis set.

\begin{tabular}{lccc}
\hline \hline State & $\mu($ a.u $)$ & $\mathrm{R}^{2}($ a.u $)$ & $\mathrm{n}_{u, n l}$ \\
\hline \hline EOM-SF-CCSD & & & \\
\hline$\tilde{X}^{3} \mathrm{~B}_{1}$ & 0.239 & 24.24 & 2.00 \\
$\tilde{a}^{1} \mathrm{~A}_{1}$ & 0.692 & 25.07 & 0.07 \\
$\tilde{b}^{1} \mathrm{~B}_{1}$ & 0.275 & 24.84 & 2.00 \\
$\tilde{c}^{1} \mathrm{~A}_{1}$ & 0.099 & 25.41 & 1.96 \\
\hline EOM-DEA-CCSD & & & \\
\hline$\tilde{X}^{3} \mathrm{~B}_{1}$ & 0.327 & 23.96 & 2.00 \\
$\tilde{a}^{1} \mathrm{~A}_{1}$ & 0.773 & 24.84 & 0.06 \\
$\tilde{b}^{1} \mathrm{~B}_{1}$ & 0.327 & 24.67 & 2.00 \\
$\tilde{c}^{1} \mathrm{~A}_{1}$ & 0.105 & 24.74 & 1.96 \\
\hline \hline
\end{tabular}

but the error for the $\tilde{c}^{1} \mathrm{~A}_{1}$ state is off by $\sim 1 \mathrm{eV}$ because of its doubly excited character. As one can see, the EOM-SFCCSD and EOM-SF-OOCCD ansätze yield excellent values for all three gaps (within $0.03 \mathrm{eV}$ from FCI) and the inclusion of triples correction reduces the errors relative to FCI to $0.01 \mathrm{eV}$ range. The EOM-DEA-CCSD performs similarly to EOM-SF-CCSD, but the errors are slightly larger $(0.002$, 0.081 , and $0.156 \mathrm{eV}$ ). This slightly worse performance can be attributed to orbital relaxation effects- the EOM-SF calculation uses the triplet-state orbitals, which are nearly optimal for all four states from the diradical manifold whereas EOMDEA-CCSD uses dication orbitals. Consequently, the $R_{2}$ operator in EOM-SF-CCSD ansatz can be fully employed to describe the differential correlation of the EOM states whereas in EOM-DEA-CCSD $R_{2}$ needs to deal with both correlation and orbital relaxation effects.

Table II compares EOM-SF-CCSD and EOM-DEA-CCSD wave functions. We observe small but noticeable differences in permanent dipole moments and the size of electron density distribution. The EOM-DEA-CCSD $<R^{2}>$ values appear to be somewhat smaller than EOM-SF-CCSD ones, which is consistent with using more compact dicationic orbitals.

Overall, despite slightly larger errors than in EOM-SFCCSD, EOM-DEA-CCSD performs rather well and is free from spin-contamination, as it relies on the closed-shell reference. We anticipate that performance of EOM-DEA-CCSD can be brought up to sub-kcal/mol range by perturbative account of higher excitations (i.e., $4 p 2 h$ ).

In addition, EOM-DEA-CCSD can describe higher excited states, which are not accessible by EOM-SF-CCSD, as was illustrated in recent studies of Rydberg diradicals ${ }^{42,43}$, a class of molecules in which the two unpaired electrons reside in two diffuse orbitals. These exotic species came into a spotlight due to their potential utility in quantum information science $^{42,43,60}$. In the context of laser cooling, which is an essential step in utilizing these molecules in quantum information applications, one needs to be able to describe not only the low-lying diradical states (as those discussed above), but also higher excited states, through which optical cycling is carried out. Hence, EOM-DEA-CCSD offers an advantage over EOM-SF-CCSD.

\section{Benzynes}

Benzynes, $\sigma \sigma$ aromatic diradicals, are popular benchmark systems for theory ${ }^{39,41,61-65}$, owing to the availability of the high-quality experimental data ${ }^{66,67}$. Table III shows adiabatic singlet-triplet gaps in ortho-, meta-, and para-benzynes; the frontier NOs and their occupations are shown in Fig. 2. The results show an anticipated trend: as the distance between the diradical centers increases, the gap shrinks and the diradical character increases, as evidenced by the occupations of the two frontier NOs in the ground singlet state. As in the methylene example, both EOM-SF-CCSD and EOM-DEA-CCSD yield accurate gaps for all three isomers. The differences between the two methods do not exceed $0.05 \mathrm{eV}$. The character of the wave functions, e.g., as illustrated by the number of effectively unpaired electrons, is also very similar.

TABLE III. Adiabatic singlet-triplet gaps (eV) in benzynes (no ZPE); cc-pVTZ basis set.

\begin{tabular}{llll}
\hline \hline Method & $o$-benzyne & $m$-benzyne & $p$-benzyne \\
\hline \hline EOM-SF-CCSD $^{a}$ & 1.578 & 0.782 & 0.147 \\
EOM-SF-CCSD(fT) $^{a}$ & 1.615 & 0.875 & 0.169 \\
EOM-SF-CCSD(dT) $^{a}$ & 1.619 & 0.892 & 0.172 \\
EOM-DEA-CCSD $^{b}$ & 1.625 & 0.799 & 0.145 \\
$\Delta$ ZPE $^{d}$ & -0.028 & 0.043 & 0.021 \\
Expt. - $\Delta$ ZPE & 1.656 & 0.868 & 0.144 \\
\hline \hline
\end{tabular}

${ }^{a}$ From Ref. 41. ${ }^{b}$ This work. ${ }^{c}$ From Ref. 66,67. ${ }^{d}$ From Ref. 39.
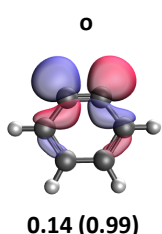

$0.11(0.98)$

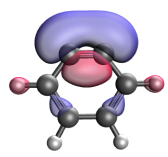

$1.85(1.00)$

$1.86(0.98)$

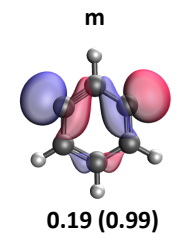

$0.18(0.98)$

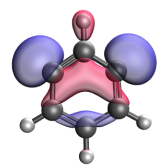

$1.80(1.00)$
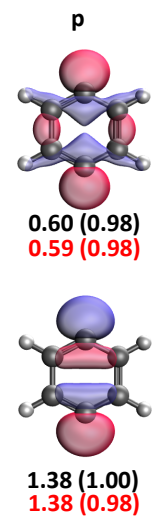

FIG. 2. Benzynes. Frontier NOs and their occupation numbers in the lowest singlet and triplet states (triplet-state occupations are given in parenthesis) computed for the EOM-SF-CCSD (black) and EOMDEA-CCSD (red) wave functions using the cc-pVTZ basis set. 
TABLE IV. Head-Gordon index $\left(n_{u, n l}\right)$ for the lowest singlet and triplet states in benzynes computed using the EOM-DEA-CCSD and EOM-SF-CCSD (numbers in parenthesis) wave functions; cc-pVTZ basis set.

\begin{tabular}{lll}
\hline \hline Type & Singlet & Triplet \\
\hline \hline$o$-benzyne & $0.11(0.16)$ & $2.00(2.00)$ \\
$m$-benzyne & $0.26(0.26)$ & $2.00(2.00)$ \\
$p$-benzyne & $1.43(1.45)$ & $2.00(2.00)$ \\
\hline \hline
\end{tabular}

\section{Cyclobutadiene}

Cyclobutadiene is a popular benchmark system ${ }^{23}$. At square $\left(\mathrm{D}_{4 h}\right)$ structures, symmetry requires that the two frontier orbitals be exactly degenerate, giving rise to a perfect diradical pattern, whereas symmetry lowering to $D_{2 h}$ lifts the degeneracy and results in a closed-shell pattern. The lowest electronic state is a singlet state. Due to the secondorder Jahn-Teller effect, the equilibrium ground state structure is rectangular $\left(D_{2 h}\right)$, with alternating double and single bonds. The lowest triplet state is not affected by the JahnTeller effect; its equilibrium geometry is $\mathrm{D}_{4 h}$. Fig. 3 shows the geometries of the $1^{3} \mathrm{~A}_{2 g}$ and $\mathrm{X}^{1} \mathrm{~A}_{g}$ states optimized at the $\operatorname{CCSD}(\mathrm{T}) / \mathrm{cc}-\mathrm{pVTZ}$ level of theory.

The ground singlet state of cyclobutadiene shows variable extent of the diradical character, which also affects low-lying electronic states. Table $\mathrm{V}$ shows the total energy for $\tilde{X}^{1} \mathrm{~A}_{g}$ ground state and vertical excitation energies for the $1^{3} \mathrm{~B}_{1 g}$, $1^{1} \mathrm{~B}_{1 g}$, and $2^{1} \mathrm{~A}_{g}$ computed with EOM-DEA-CCSD and other methods. Table VI shows the total energy for the $\tilde{X}^{1} \mathrm{~B}_{1 g}$ ground state and vertical excitation energies for $1^{3} \mathrm{~A}_{2 g}, 2^{1} \mathrm{~A}_{1 g}$, and $1^{1} \mathrm{~B}_{2 g}$ for EOM-DEA-CCSD and other methods.
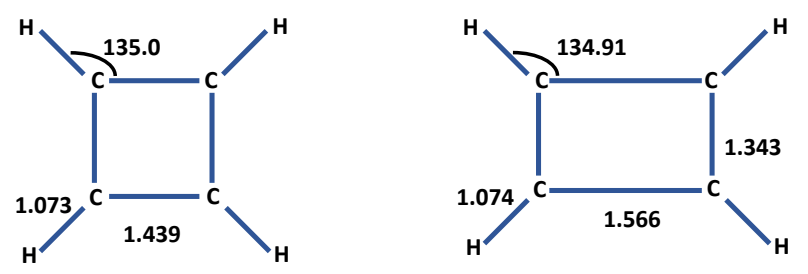

FIG. 3. Geometries of the $1^{3} \mathrm{~A}_{2 g}$ (left) and $\mathrm{X}^{1} \mathrm{~A}_{g}$ (right) states optimized at the $\operatorname{CCSD}(\mathrm{T}) / \mathrm{cc}-\mathrm{pVTZ}$ level of theory. Bond lengths are in angstroms and angles are in degrees; the structures are from Ref. 11.

Total EOM-DEA-CCSD energies for the ground state are significantly above EOM-SF-CCSD ones, by about $\sim 0.02$ hartree, which we attribute to using dication orbitals in the former. However, the differences in the respective excitation energies are small. At both geometries $\left(\mathrm{D}_{4 h}\right.$ and $\mathrm{D}_{2 h}$ structures) the vertical excitation energies obtained with EOM-DEA-CCSD are underestimated relative to the reference UHF/ROHF-EOM-SF-CCSD(dT) values; this can also be attributed to the use of compact Hartree-Fock orbitals of
TABLE V. Total energies (hartree) of the ground state $X^{1} \mathrm{~A}_{g}$ of cyclobutadiene and vertical excitation energies $(\mathrm{eV})$ at the $X^{1} \mathrm{~A}_{g}$ equilibrium geometry ( $\mathrm{D}_{2 h}$ symmetry, 4 frozen core orbitals); cc-pVTZ basis set.

\begin{tabular}{lcccc}
\hline \hline Method & $E_{t o t}\left(\mathrm{X}^{1} \mathrm{~A}_{g}\right)$ & $1^{3} \mathrm{~B}_{1 g}$ & $1^{1} \mathrm{~B}_{1 g}$ & $2^{1} \mathrm{~A}_{g}$ \\
\hline \hline EOM-EE-CCSD & -154.35495 & 1.349 & 3.314 & 7.874 \\
EOM-EE-CCSDT & -154.39067 & - & 3.264 & 4.512 \\
UHF-EOM-SF-CCSD & -154.36285 & 1.652 & 3.411 & 4.354 \\
UHF-EOM-SF-CCSD(fT) & -154.36745 & 1.516 & 3.257 & 4.203 \\
UHF-EOM-SF-CCSD(dT) & -154.36744 & 1.474 & 3.210 & 4.174 \\
ROHF-EOM-SF-CCSD & -154.36339 & 1.656 & 3.408 & 4.348 \\
ROHF-EOM-SF-CCSD(fT) & -154.36756 & 1.515 & 3.253 & 4.197 \\
ROHF-EOM-SF-CCSD(dT) & -154.36737 & 1.467 & 3.200 & 4.168 \\
EOM-DEA-CCSD & -154.33922 & 1.403 & 3.120 & 4.127 \\
\hline \hline
\end{tabular}

TABLE VI. Total energies (hartree) of the ground state $\mathrm{X}^{1} \mathrm{~B}_{1 g}$ of cyclobutadiene and vertical excitation energies $(\mathrm{eV})$ at the $\mathrm{X}^{1} \mathrm{~B}_{1 g}$ equilibrium geometry ( $\mathrm{D}_{4 h}$ symmetry); cc-pVTZ basis set

\begin{tabular}{lcccc}
\hline \hline Method & $E_{\text {tot }}\left(\mathrm{X}^{1} \mathrm{~B}_{1 g}\right)$ & $1^{3} \mathrm{~A}_{2 g}$ & $2^{1} \mathrm{~A}_{1 g}$ & $1^{1} \mathrm{~B}_{2 g}$ \\
\hline \hline EOM-EE-CCSD $^{a}$ & -154.38058 & -0.590 & - & 1.534 \\
UHF-EOM-SF-CCSD $^{a}$ & -154.41301 & 0.369 & 1.824 & 2.143 \\
UHF-EOM-SF-CCSD $(\mathrm{fT})^{a}$ & -154.41478 & 0.163 & 1.530 & 1.921 \\
UHF-EOM-SF-CCSD $(d T)^{b}$ & -154.41390 & 0.098 & 1.456 & 1.853 \\
ROHF-EOM-SF-CCSD $^{a}$ & -154.41342 & 0.369 & 1.814 & 2.137 \\
${\text { ROHF-EOM-SF-CCSD }(\mathrm{fT})^{b}}^{b}$ & -154.41477 & 0.159 & 1.521 & 1.915 \\
${\text { ROHF-EOM-SF-CCSD }(\mathrm{dT})^{b}}^{b}$ & -154.41358 & 0.088 & 1.438 & 1.837 \\
EOM-DEA-CCSD $^{c}$ & -154.38600 & 0.023 & 1.406 & 1.751 \\
\hline \hline
\end{tabular}

${ }^{a}$ From Ref. 11. ${ }^{b}$ From Ref. 41. ${ }^{c}$ This work.

the +2 reference state. However, EOM-DEA-CCSD is closer to UHF/ROHF-EOM-SF-CCSD(dT) than EOM-EE-CCSD or EOM-SF-CCSD.

\section{E. Ozone}

Ozone has been extensively studied because of its role in atmospheric chemistry ${ }^{58,68}$. Owing to its non-classical bonding pattern, which cannot be described by a single Lewis structure, ozone features the ground state of diradical character and low-lying excited states.

Ozone has 24 electrons and belongs to $\mathrm{C}_{2 v}$ symmetry. Electronic configurations of its low-lying states are summarized in Table VII and in Fig. 4, which shows relevant frontier NOs. Table VII lists the occupation numbers of the leading configurations in the ground and several excited states. The [core] denotes 9 molecular orbitals that are doubly occupied in ground state and in the excited state discussed here. Electronic configuration of ozone's ground state is [core] ${ }^{18}\left(4 b_{2}\right)^{2}\left(6 a_{1}\right)^{2}\left(1 a_{2}\right)^{2}$. To compute the ground and excited states of ozone by EOMDEA-CCSD, we need to use a +2 charge reference. One can consider different choices, e.g., REF1=[core $]^{18}\left(4 b_{2}\right)^{2}\left(1 a_{2}\right)^{2}$ and REF2 $=[\text { core }]^{18}\left(4 b_{2}\right)^{2}\left(6 a_{1}\right)^{2}$. Both references are suitable for describing the ground state, but the accessibility of excited states varies. When starting from REF $1, \mathrm{X}^{1} \mathrm{~A}_{1},{ }^{3} \mathrm{~B}_{1}$, ${ }^{1} \mathrm{~B}_{1}$, and $2^{1} \mathrm{~A}_{1}$ excited states can be described accurately, be- 
cause their leading electronic configuration can be generated by $2 p$ part of the EOM-DEA operator. In contrast, when using REF2, only $\mathrm{X}^{1} \mathrm{~A}_{1}$ and ${ }^{3} \mathrm{~B}_{2}$ can be described accurately. Neither REF1 nor REF2 allows access to the ${ }^{1} \mathrm{~A}_{2}$ excited state, because from either one one requires $3 h 1 p$ DEA operators.

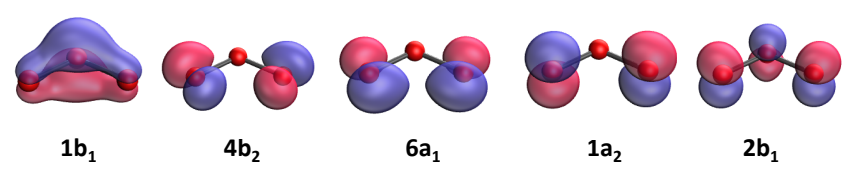

FIG. 4. Frontier natural orbitals of the $\mathrm{X}^{1} \mathrm{~A}_{1}$ state of ozone. computed with EOM-DEA-CCSD/aug-cc-pVTZ. In the ground state, $2 b_{1}$ orbital is vacant.

TABLE VII. Orbital occupation in dominant configurations of the ground and low-lying excited states in ozone

\begin{tabular}{ccccccc}
\hline \multicolumn{5}{c}{ Configurations } \\
\cline { 2 - 7 }
\end{tabular}

Table VIII compares vertical excitation energies in ozone computed with EOM-EE-CCSD and EOM-DEA-CCSD using the aug-cc-pVTZ basis set. Relative to EOM-EE-CCSD, EOM-DEA-CCSD underestimates the excitation energy of the ${ }^{1} B_{1}$ state, whereas the excitation energies of the ${ }^{3} B_{2}$ and ${ }^{3} \mathrm{~B}_{1}$ states are overestimated. The advantage of EOM-DEA$\mathrm{CCSD}$ is that it captures the doubly excited state, $2^{1} \mathrm{~A}_{1}$. However, it cannot access the ${ }^{3} \mathrm{~A}_{2}$ state.

TABLE VIII. Ozone. Vertical $^{a}$ excitation energies (eV) relative to the $\mathrm{X}^{1} A_{1}$ state computed by EOM-EE-CCSD and EOM-DEACCSD; aug-cc-pVTZ

\begin{tabular}{cccc}
\hline \hline State & EE-CCSD $^{b}$ & DEA-CCSD $^{c}$ & DEA-CCSD $^{d}$ \\
\hline \hline${ }^{3} \mathrm{~B}_{2}$ & 1.303 & - & 1.60 \\
${ }^{3} \mathrm{~B}_{1}$ & 1.701 & 1.956 & - \\
${ }^{3} \mathrm{~A}_{2}$ & 1.868 & - & - \\
${ }^{1} \mathrm{~A}_{2}$ & 2.279 & - & - \\
${ }^{1} \mathrm{~B}_{1}$ & 2.307 & 2.262 & - \\
$2^{1} \mathrm{~A}_{1}$ & - & 4.99 & - \\
\hline \hline
\end{tabular}

$a \overline{\text { Geometry used: } r_{O O}=1.2724 \AA, \theta_{O O O}=116.82^{\circ}}$

${ }^{b} \mathrm{HF}$ reference $=[\text { core }]^{18}\left(4 b_{2}\right)^{2}\left(6 a_{1}\right)^{2}\left(1 a_{2}\right)^{2}$

${ }^{c}$ HF reference $=[\text { core }]^{18}\left(4 b_{2}\right)^{2}\left(1 a_{2}\right)^{2}$

${ }^{d}$ HF reference $=[\text { core }]^{18}\left(4 b_{2}\right)^{2}\left(6 a_{1}\right)^{2}$
Several theoretical studies have discussed the existence of two stable ground-state structures for ozone ${ }^{69,70}$ : the familiar open-triangle structure and a closed-ring structure (both have $\mathrm{C}_{2 v}$ symmetry). Fig. 5 shows the geometries of open-ring minimum (OM), the hypothetical ring minimum (RM), and the transition state (TS) between them, taken from Ref. 70. Table IX shows the energy gaps between two lowest singlet states of ozone for these three geometries. The lowest $X^{1} A_{1}$ state in RM and TS structures lies $2.86 \mathrm{eV}$ and $1.50 \mathrm{eV}$ above the OM geometry. As one can see, EOM-DEA-CCSD agrees reasonably well with the order of states at different geometries as compared to energies from Ref. 70.

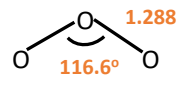

OM

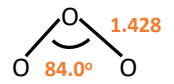

TS

$1^{1} \mathrm{~A}_{1} \overline{0.00 \mathrm{eV}}$

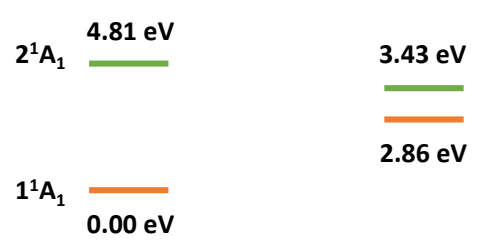

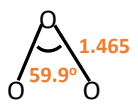

RM

$9.50 \mathrm{eV}$
FIG. 5. Ozone. Energies of the two lowest singlet states at selected structures; EOM-DEA-CCSD/cc-pVTZ.

TABLE IX. Ozone. Energy gap (eV) between the $2^{1} A_{1}$ and $1^{1} A_{1}$ states computed with extrapolated semistochastic heat-bath configuration interaction (ex. SHCI), MRCI(SDTQ), and EOM-DEACCSD; cc-pVTZ basis set.

\begin{tabular}{lccc}
\hline \hline Geometry & ex. SHCI $^{a}$ & MRCI(SDTQ) $^{b}$ & DEA-CCSD $^{c}$ \\
\hline \hline OM & 4.13 & $3.54-4.63$ & 4.77 \\
RM & 0.01 & $0.05-0.16$ & 0.60 \\
TM & 6.13 & $7.35-8.44$ & 8.02 \\
\hline \hline \multicolumn{4}{c}{${ }^{a}$ Ref. 70. ${ }^{b}$ Ref. 71. ${ }^{c}$ This work. }
\end{tabular}

\section{F. Ethylene}

Table X shows the ground-state energy of ethylene along the torsional coordinate computed with different methods; the respective torsion barriers are summarized in Table XII. Table XIII compares vertical excitation energies for relevant excited states of ethylene at its equilibrium geometry $\left(0^{\circ}\right.$ torsion angle) computed with EOM-EE-CCSD and EOM-DEA-CCSD using the aug-cc-pVTZ basis set.

At the twisted geometries, the overlap between the two $p$ orbitals is reduced and the gap between $\pi$ and $\pi^{*}$ shrinks. At $90^{\circ}, \pi$ and $\pi^{*}$ should be exactly degenerate and the double bond is broken. In the closed-shell Hartree-Fock reference, 
TABLE X. Ethylene torsion, DZP basis. Total energies (hartree) for the SF-TDDFT(5050), TCSCF-CISD, CCSD, EOM-SF-CCSD, and EOM-DEA-CCSD models.

\begin{tabular}{ccccccc}
\hline \hline$\Theta$ & SF-5050 & TCSCF-CISD & CCSD & EOM-SF-CCSD/UHF & EOM-DEA/+2 & EOM-DEA/0 \\
\hline \hline 0 & -78.53417 & -78.36589 & -78.35221 & -78.35643 & -78.34170 & -78.37023 \\
15 & -78.52957 & -78.36143 & -78.34773 & -78.35199 & -78.33690 & -78.36415 \\
30 & -78.51596 & -78.34812 & -78.33434 & -78.33880 & -78.32367 & -78.34881 \\
45 & -78.49369 & -78.32634 & -78.31223 & -78.31724 & -78.30241 & -78.32582 \\
60 & -78.46365 & -78.29724 & -78.28205 & -78.28850 & -78.24240 & -78.26383 \\
75 & -78.42882 & -78.26471 & -78.24550 & -78.25646 & -78.23321 & -78.25447 \\
80 & -78.41799 & -78.25522 & -78.23262 & -78.24715 & -78.22656 & -78.24767 \\
85 & -78.40965 & -78.24833 & -78.22002 & -78.24039 & -78.22407 & -78.22006 \\
90 & -78.40634 & -78.24574 & -78.20851 & -78.23785 & -1160. & \\
\hline \hline
\end{tabular}

Geometry used: $r_{C C}=1.330 \AA, r_{C-H}=1.076 \AA$, and $\theta_{H C H}=116^{\circ}$.

the two orbitals are not treated in a balanced way, which results in a high barrier and a cusp on the torsional potential. Inclusion of the correlation in the CCSD ansatz reduces the barrier height, but cannot fully eliminate the cusp, because of an unbalanced treatment of $(\pi)^{2}$ and $\left(\pi^{*}\right)^{2}$ configurations. In contrast, EOM-SF-CCSD (with a high-spin triplet reference) and EOM-DEA-CCSD (with a dication reference) are treating the two frontier orbitals and the respective configurations in a balanced way, yielding smooth potential energy curves and accurate barrier heights (as compared to the reference MRCI values).

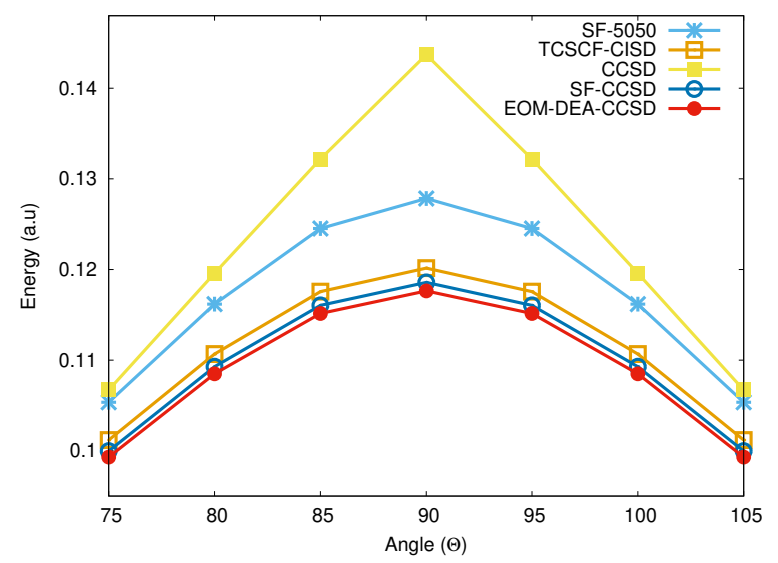

FIG. 6. Ethylene torsion barrier computed with various methods and a DZP basis set. All curves are shifted such that the energy at $0^{0}$ is zero

We note that the cusp reappears in the EOM-DEA-CCSD calculations that use the neutral reference orbitals. These calculations also overestimate the barrier height (i.e., the difference between +2 and 0 calculation is $0.89 \mathrm{eV}$ ). The analysis of the relevant orbitals (shown in the SI) attributes this to the scrambling of the $\pi$ HOMO with the low-lying orbitals (in the neutral reference), leading to symmetry breaking.

Table XIII and Table XIV compares excitation energies and one-particle properties computed using EOM-DEA-CCSD and EOM-EE-CCSD wavefunctions. As expected, EOM-

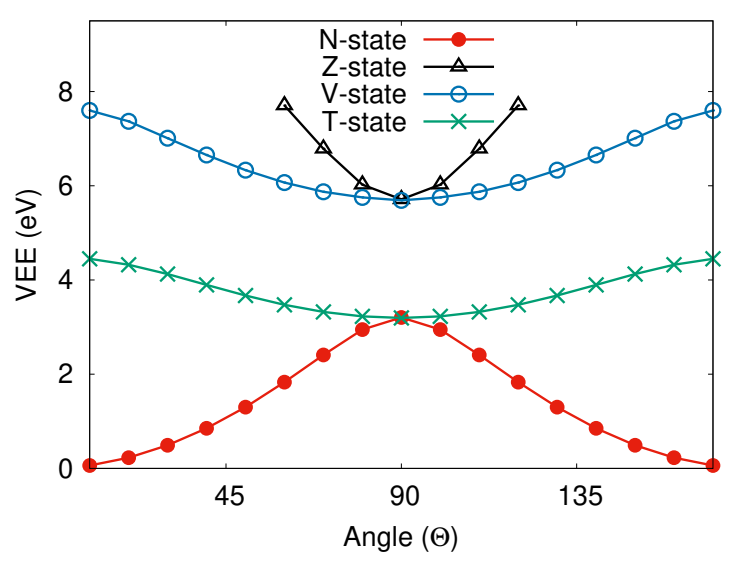

FIG. 7. Potential energy curves along torsion coordinate for several electronic states of ethylene computed with EOM-DEA-CCSD/augcc-pVTZ.

TABLE XI. Ethylene. Vertical excitation energies $(\mathrm{eV})$ from the lowest singlet state $\left({ }^{1} \mathrm{~A}_{2}\right)$ at the twisted geometry using EOM-DEACCSD/aug-cc-pVTZ.

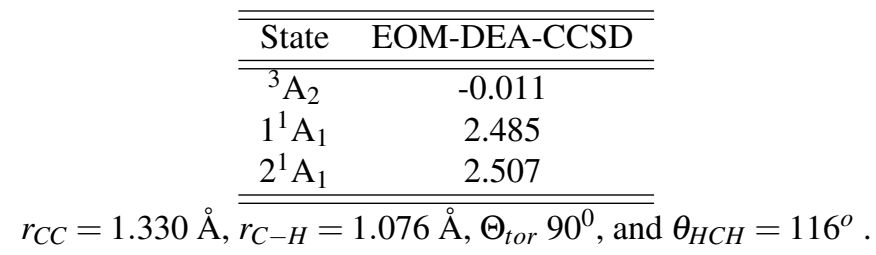

DEA-CCSD excitation energies are comparable to the EOMEE-CCSD ones for all excited states that are dominated by one-electron excitation. EOM-DEA-CCSD can also describe doubly excited state, ${ }^{1} \mathrm{~A}_{g}$, which is not accessible by EOMEE-CCSD. 
TABLE XII. Ethylene torsion barrier (eV) computed with various methods and a DZP basis set.

\begin{tabular}{lc}
\hline \hline Method & Barrier \\
\hline \hline SF-TDDFT/5050 & 3.48 \\
TCSCF-CISD & 3.27 \\
CCSD & 3.91 \\
EOM-SF-CCSD/UHF & 3.23 \\
EOM-DEA-CCSD/+2 & 3.20 \\
EOM-DEA-CCSD/+0 & 4.09 \\
EOM-DEA-CCSD $^{* *} /+0$ & 3.41 \\
\hline \hline
\end{tabular}

${ }^{* *}$ Dihedral angle $=89.9999^{\circ}$.

This allows the symmetry of molecule remain in $D_{2}$ point group.

TABLE XIII. Ethylene. Vertical excitation energies (eV) at the equilibrium geometry using EOM-DEA-CCSD with aug-cc-pVTZ.

\begin{tabular}{|c|c|c|c|c|}
\hline State & Orbital assign & Exp. & DEA-CCSD & EE-CCSD \\
\hline${ }^{3} \mathrm{~B}_{1 u}$ & ${ }^{3}\left(\pi, \pi^{*}\right)$ & 4.36 & 4.48 & 4.50 \\
\hline${ }^{3} \mathrm{~B}_{3 u}$ & $3(\pi, 3 s)$ & 6.98 & 6.82 & 7.31 \\
\hline${ }^{1} \mathrm{~B}_{3 u}$ & ${ }^{1}(\pi, 3 s)$ & 7.11 & 6.92 & 7.44 \\
\hline${ }^{1} \mathrm{~B}_{1 u}$ & ${ }^{1}\left(\pi, \pi^{*}\right)$ & $7.68(8.0)$ & 7.79 & 8.04 \\
\hline${ }^{2} \mathrm{~B}_{3 u}$ & ${ }^{2}(\pi)$ & 10.5 & 10.43 & 10.43 \\
\hline${ }^{1} \mathrm{~A}_{g}$ & ${ }^{1}\left(\pi^{*}, \pi^{*}\right)$ & - & 12.86 & - \\
\hline
\end{tabular}

TABLE XIV. One-particle state and transition properties for ethylene computed with EOM-EE-CCSD and EOM-DEA-CCSD; augcc-pVTZ basis set.

\begin{tabular}{cccccc}
\hline \hline State & $\mathrm{n}_{u, n l}$ & $f_{l}$ & $\|\gamma\|$ & $Z_{H E}$ & e-h sep \\
\hline \hline EOM-EE-CCSD & & & & & \\
\hline${ }^{3} \mathrm{~B}_{1 u}$ & 2.00 & 0.00 & 0.94 & 1.39 & 1.82 \\
${ }^{3} \mathrm{~B}_{3 u}$ & 2.00 & 0.00 & 0.91 & 1.03 & 3.60 \\
${ }^{1} \mathrm{~B}_{3 u}$ & 2.00 & 0.08 & 0.91 & 1.01 & 3.71 \\
${ }^{1} \mathrm{~B}_{1 u}$ & 2.00 & 0.37 & 0.92 & 1.80 & 2.59 \\
${ }^{1} \mathrm{~A}_{g}$ & - & - & - & - & - \\
\hline EOM-DEA-CCSD & & & & & \\
\hline${ }^{3} \mathrm{~B}_{1 u}$ & 2.00 & 0.00 & 0.91 & 1.37 & 1.87 \\
${ }^{3} \mathrm{~B}_{3 u}$ & 2.00 & 0.00 & 0.95 & 1.02 & 3.64 \\
${ }^{1} \mathrm{~B}_{3 u}$ & 2.00 & 0.09 & 0.91 & 1.01 & 3.74 \\
${ }^{1} \mathrm{~B}_{1 u}$ & 2.00 & 0.38 & 0.93 & 1.41 & 2.81 \\
${ }^{1} \mathrm{~A}_{g}$ & 0.55 & - & - & - & - \\
\hline
\end{tabular}

\section{G. Butadiene}

Butadiene is the smallest polyene, representing an important motif commonly occurring in photoactive molecules and dyes. The theoretical description of the two lowest states in polyenes is challenging due to their different character. In butadiene, the lowest excited state, $1^{1} \mathrm{~B}_{u}^{+}$, is a bright, dipoleallowed, singly excited state. The second excited state, $2^{1} \mathrm{~A}_{g}^{-}$ is dark, dipole-forbidden state, which is believed to have substantial doubly excited character. UV-VIS absorption spectrum of butadiene places the $1^{1} \mathrm{~B}_{u}^{+}$state at $5.92 \mathrm{eV}$ above the ground state (vertically). Because butadiene is able to fluoresce, the consensus is that the dark state is located above the bright state. In longer polyenes, the dark state drops below the bright state, which leads to fluorescence quenching. The exact positions, and even the ordering, of the two states in short polyenes, butadiene and hexatriene, has been debated, as summarized, for example, in Ref. 56. The difficulties in resolving this issue theoretically stem from the strong dynamical correlation effects in the bright state and some doubly excited character of the dark state. Only high-level methods, such as EOM-EE-CCSDT, can provide an accurate description of these two effects. Moreover, because of the contributions of Rydberg excitations into the dark state, the results (including wave function composition) are sensitive to the basis set. Consequently, the results of approximate treatments vary widely. Multi-reference methods tend to overestimate the doubly excited character of the dark state and underestimate the contributions from Rydberg excitations. In contrast, single-reference methods, such as EOM-CCSD or ADC, underestimate doubly excited contributions in the dark state.

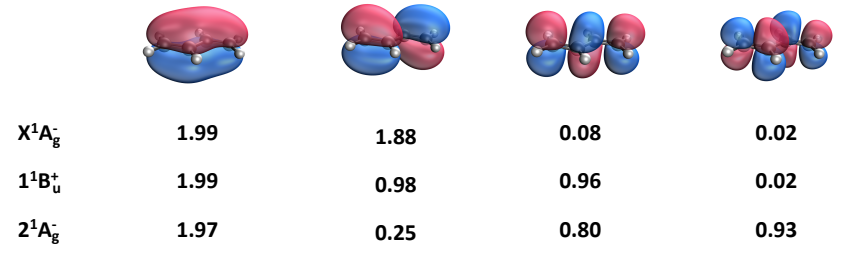

FIG. 8. Butadiene. Natural frontier orbitals and their occupations computed using the EOM-DEA-CCSD/aug-cc-pVTZ wave functions (using dication reference orbitals).

Our results show that the EOM-DEA-CCSD ansatz is capable of describing both states on the same footing, yielding excitation energies of the two states in good agreement with the reference EOM-EE-CCSDT values. This is illustrated by the data in Table XV, which compares excitation energies for the bright $\left(1^{1} \mathrm{~B}_{u}^{+}\right)$and dark $\left(2^{1} \mathrm{~A}_{g}^{-}\right)$excited states of transbutadiene computed with the EOM-EE-CCSD, EOM-DEACCSD, EOM-EE-CC3, and EOM-EE-CCSDT methods with different basis sets. For EOM-DEA-CCSD, we report the results obtained with two references: one constructed using the default dication orbitals and one constructed using the orbitals from the neutral system. Excitation energies for both states using EOM-DEA-CCSD are comparable to EOM-EE-CCSDT and EOM-EE-CC3.

For the bright state, the effect of triple excitations is small and the difference between EOM-EE-CCSD and EOM-EECCSDT is $\sim 0.1 \mathrm{eV}$; as usual, inclusion of triples brings the excitation energies down. The EOM-DEA-CCSD values are slightly below EOM-EE-CCSD and, therefore, are slightly closer to EOM-EE-CCSDT. Using neutral orbitals increases the excitation energy, which can be attributed to better description of the ground state. The effect of the basis set is 
TABLE XV. Vertical excitation energies $(\mathrm{eV})$ for the $1{ }^{1} \mathrm{~B}_{u}^{+}$and $2^{1} \mathrm{~A}_{g}^{-}$in butadiene

\begin{tabular}{lcccccccccc}
\hline \hline \multirow{2}{*}{ Basis } & \multicolumn{2}{c}{ EE-CCSD $^{a}$} & \multicolumn{2}{c}{ DEA-CCSD/+2 } & \multicolumn{2}{c}{ DEA-CCSD/0 } & \multicolumn{2}{c}{ EE-CC3 $^{b}$} & \multicolumn{2}{c}{ EE-CCSDT $^{b}$} \\
& $1^{1} \mathrm{~B}_{u}^{+}$ & $2^{1} \mathrm{~A}_{g}^{-}$ & $1^{1} \mathrm{~B}_{u}^{+}$ & $2^{1} \mathrm{~A}_{g}^{-}$ & $1^{1} \mathrm{~B}_{u}^{+}$ & $2^{1} \mathrm{~A}_{g}^{-}$ & $1^{1} \mathrm{~B}_{u}^{+}$ & $2^{1} \mathrm{~A}_{g}^{-}$ & $1^{1} \mathrm{~B}_{u}^{+}$ & $2^{1} \mathrm{~A}_{g}^{-}$ \\
\hline \hline cc-pVDZ & 6.918 & 7.648 & 6.893 & 7.412 & 6.857 & 7.449 & 6.776 & 6.968 & 6.794 & 6.830 \\
cc-pVTZ & 6.660 & 7.555 & 6.643 & 7.206 & 6.632 & 7.296 & 6.514 & 6.870 & 6.535 & 6.763 \\
cc-pVQZ & 6.562 & 7.458 & 6.520 & 7.074 & - & - & - & - & - & 6.722 \\
aug-cc-pVDZ & 6.389 & 7.057 & 6.265 & 6.684 & 6.353 & 6.867 & 6.269 & 6.661 & 6.285 & 6.577 \\
aug-cc-pVTZ & 6.365 & 7.093 & 6.241 & 6.679 & 6.343 & 6.883 & 6.238 & 6.654 & $6.241^{c}$ & - \\
\hline \hline
\end{tabular}

${ }^{a}$ Ref. 56. ${ }^{b}$ This work. ${ }^{c}$ Estimated with nine frozen orbitals and extrapolation ${ }^{56}$.

noticeable - including diffuse functions lowers the excitation energy of the bright state by $0.2 \mathrm{eV}$.

The results for $2^{1} \mathrm{~A}_{g}^{-}$show larger differences between EOM-EE-CCSD and EOM-EE-CCSDT. In the small basis set (cc-pVDZ), the difference is $0.8 \mathrm{eV}$, but it shrinks to 0.3 $\mathrm{eV}$ when diffuse functions are included. This illustrates the importance of Rydberg contributions and that the effect of double excitations is exaggerated when using compact basis sets (this is why most multi-reference calculations significantly overestimate doubly excited character of the dark state). EOM-DEA-CCSD energies for the dark state are closer to EOM-EE-CCSDT, yielding smaller errors than those of EOM-EE-CCSD. In aug-cc-pVDZ basis, the energy of the dark state is overestimated by only $0.1 \mathrm{eV}$ by EOM-DEACCSD.The $\mathrm{CC} 3$ results show similar trends.

These observations are supported by the wave function analysis of the EOM-EE-CCSD, EOM-EE-CC3, and EOMDEA-CCSD wave functions, summarized in Table XVI (see also Table S1 in the SI). Fig. 8 shows the occupancy of natural orbitals for ground and two excited states (described by EOM-DEA-CCSD with dicationic reference). Occupations of the frontier NOs clearly reveal singly excited character of the $1^{1} \mathrm{~B}_{u}^{+}$state and contributions from doubly excited configurations in the $2^{1} \mathrm{~A}_{g}^{-}$state. The comparison of $\|\gamma\|$ computed with EOM-EE-CCSD,CC3, and EOM-DEACCSD shows that doubly excited character increases (in CC3 and EOM-DEA-CCSD relative to EOM-EE-CCSD), but overall is not dominant. Similar conclusions can be drawn from the values of $R_{2}^{2}$ (collected in Table $\mathrm{S} 1$ in the SI). The comparison between the regular and augmented basis sets also show that the doubly excited character is overestimated when the basis does not capture substantial Rydberg character in the $2^{1} \mathrm{~A}_{g}^{-}$state.

\section{H. Potential energy surfaces and conical intersections in retinal chromophore}

Retinal is a well studied model system ${ }^{44-46}$, featuring conical intersections between the two lowest electronic states relevant to the cis-trans photoinduced isomerization of rhodopsin. The two lowest states are the ground state and a bright $\pi \pi^{*}$ excited state. Photoexcitation changes the conjugation patter and initiates twisting, which imparts diradical character into the ground state, somewhat similar to the ethylene torsion. We
TABLE XVI. One-particle state and transition properties of butadiene computed with EOM-EE-CCSD, EOM-EE-CC3, and EOMDEA-CCSD.

\begin{tabular}{lccccc}
\hline \hline State & $\mathrm{n}_{u, n l}$ & $f_{l}$ & $\|\gamma\|$ & $Z_{H E}$ & $\mathrm{PR}_{n t o}$ \\
\hline \hline EE-CC3/cc-pVDZ & & & & & \\
\hline $1^{1} \mathrm{~B}_{u}^{+}$ & - & 0.71 & 0.86 & 1.92 & 1.12 \\
$2^{1} \mathrm{~A}_{g}^{-}$ & - & 0.00 & 0.54 & 4.19 & 2.00 \\
\hline EE-CC3/aug-cc-pVDZ & & & & & \\
\hline $1^{1} \mathrm{~B}_{u}^{+}$ & - & 0.67 & 0.85 & 1.74 & 1.09 \\
$2^{1} \mathrm{~A}_{g}^{-}$ & - & 0.00 & 0.65 & 3.31 & 1.63 \\
\hline EE-CC3/aug-cc-pVTZ & & & & & \\
\hline $1^{1} \mathrm{~B}_{u}^{+}$ & - & 0.66 & 0.85 & 1.75 & 1.09 \\
$2^{1} \mathrm{~A}_{g}^{-}$ & - & 0.00 & 0.65 & 3.39 & 1.66 \\
\hline EE-CCSD/aug-cc-pVTZ & & & & & \\
\hline $1^{1} \mathrm{~B}_{u}^{+}$ & 2.00 & 0.71 & 0.88 & 1.71 & 1.09 \\
$2^{1} \mathrm{~A}_{g}^{-}$ & 2.10 & 0.00 & 0.82 & 2.14 & 1.25 \\
\hline $\mathrm{DEA}-\mathrm{CCSD} /+2 / a u g-c c-p V T Z$ & & & & & \\
\hline $1^{1} \mathrm{~B}_{u}^{+}$ & 2.00 & 0.79 & 0.91 & 1.43 & 1.06 \\
$2^{1} \mathrm{~A}_{g}^{-}$ & 2.11 & 0.00 & 0.78 & 1.30 & 1.04 \\
\hline \hline
\end{tabular}

use this example to assess the ability of EOM-DEA-CCSD to describe conical intersections. Fig. 9 shows the PES of the lowest electronic state as function of the two key coordinates: bond-length alternation (BLA) and twining reaction coordinate $(\mathrm{RC})$. As one can see, the character of the lowest adiabatic state changes because the two lowest states exchange their character. The location of conical intersection is very sensitive to the electronic structure method, as it requires balanced description of the two electronic states. Previous benchmark studies ${ }^{44-46}$ have shown that EOM-SF-CCSD(dT) and and MR-CISD+Q results are in very good agreement, while lower-level methods show large discrepancies.

Fig. 10 shows the PES scans along the BLA coordinate. The shape of the PES of the diradical state along BLA coordinate computed by EOM-DEA-CCSD agrees well with the MR-CISD+Q results, and is not affected by using different reference orbitals $(+1$ or +3$)$. The situation is different for the charge-transfer state. Overall shape agrees with MRCISD+Q, the energy relative to the diradical state depends on the orbital choice, which results in large differences in the location of conical intersection. Using +3 Hartree-Fock orbitals in EOM-DEA-CCSD, the conical intersection appears too early along BLA coordinate, as compared with MR-CISD. 


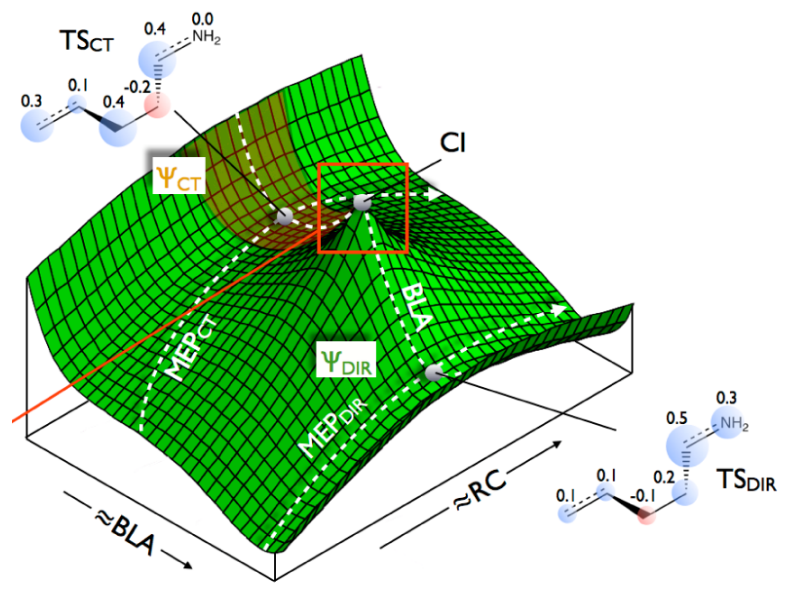

FIG. 9. Potential energy surface in retinal showing the location of the conical intersection (CoIn or CI) between the charge transfer and diradical states. The two coordinates are bond-alternation (BLA) and twisting reaction coordinate (RC). Relevant mechanistic paths are indicated as white dashed lines. $\mathrm{MEP}_{\mathrm{CT}}$ : minimum energy path on the ground state that connects the cis and trans retinal equilibrium geometries through a transition state $\left(\mathrm{TS}_{\mathrm{CT}}\right)$. $\mathrm{MEP}_{\mathrm{DIR}}$ : connects cis, $\mathrm{TS}_{\mathrm{DIR}}$ and trans structures of the $\Psi_{\mathrm{DIR}}$ state. The BLA path connects the $\mathrm{TS}_{\mathrm{CT}}$ and $\mathrm{TS}_{\mathrm{DIR}}$ transition states and also intercepts a CoIn (CI) point. Atomic charges of the two transition states are illustrated by bubble diagrams. Reproduced with permission from Ref. 44.

In contrast, when using +1 Hartree-Fock orbitals, conical intersection appears too late along the BLA coordinate, as compared to MR-CISD+Q. Due to large positive charge in $+3 \mathrm{HF}$ reference, the molecular orbital and their energy shows large deviations from +1 reference, and $3 p 1 h$ part of the EOMDEA-CCSD ansatz is not sufficient to describe both orbital relaxation and the correlation effects in the charge-transfer state. Consequently, the total energy of charge-transfer state is higher, leading to the shift in the conical intersection position. This also leads to an opposite trend in energy gap $\left(\mathrm{S}_{0}\right.$ and $\left.\mathrm{S}_{1}\right)$ at $\mathrm{TS}_{C T}$ and $\mathrm{TS}_{D I R}$, when compared with MR-CISD+Q, as shown in Table XVII.

TABLE XVII. The $\mathrm{S}_{0}$ and $\mathrm{S}_{1}$ energy gaps $(\mathrm{kcal} / \mathrm{mol})$ at $\mathrm{TS}_{C T}, \mathrm{TS}_{D I R}$, and cis-PSB3 geometry of retinal; 6-31G* basis set.

\begin{tabular}{lccc}
\hline \hline Method & TS $_{C T}$ & TS $_{D I R}$ & cis-PSB3 \\
\hline \hline MRCISD+Q & 10.2 & 0.6 & 101.4 \\
MRCISD & 8.8 & 1.6 & 104.8 \\
CASSCF & 4.5 & 7.4 & 110.3 \\
EE-CCSD & 16.6 & 6.9 & \\
SF-CCSD/UHF & 2.4 & 7.5 & \\
SF-CCSD/ROHF & 5.8 & 6.0 & 105.5 \\
SF-CCSD(dT)/ROHF & 11.1 & 0.6 & 102.1 \\
SF-CCSD(fT)/ROHF & 10.2 & 0.6 & \\
DEA-CCSD/+3 & 1.49 & 12.71 & 105.2 \\
DEA-CCSD/+1 & 15.13 & 4.39 & 104.6 \\
\hline \hline
\end{tabular}

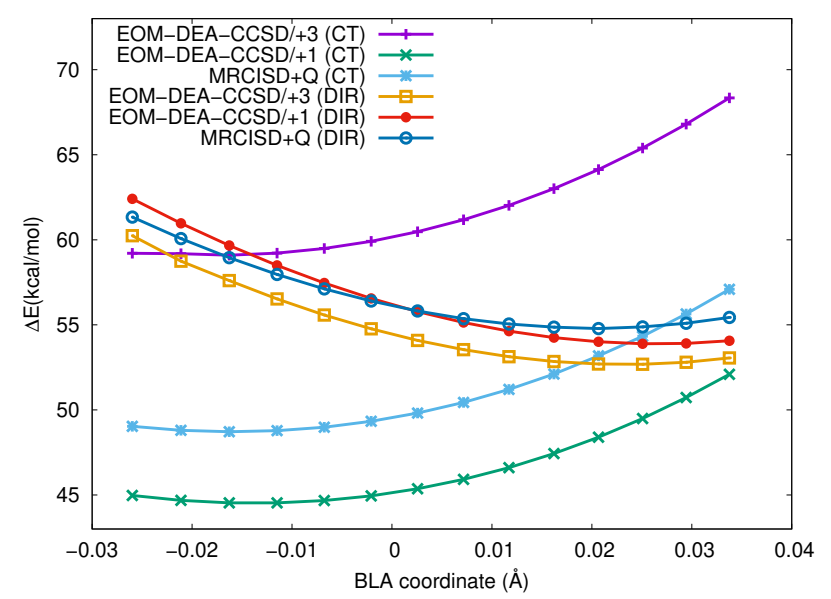

FIG. 10. The $\mathrm{S}_{0}$ and $\mathrm{S}_{1}$ energy profiles (retinal) along the BLA coordinate computed with EOM-DEA-CCSD (with orbitals from the +3 and +1 charged Hartree-Fock reference) and MRCISD+Q using the $6-31 \mathrm{G}^{*}$ basis set. The energy values are relative to cis-PSB3.

\section{Excited states in water and ammonia}

In this section we consider two small molecules (water and ammonia) from Head-Gordon's data set and assess the performance of EOM-DEA-CCSD for the singly excited states. In both molecules, the low-lying states have predominantly Rydberg character. Tables XVIII and XIX compare vertical excitation energies computed with EOM-DEA-CCSD and other methods using aug-cc-pVQZ for ammonia and water, respectively. The results show that EOM-DEA-CCSD excitation energies are generally below exFCI ones (extrapolated FCI). This can be attributed to using compact dicationic reference orbitals. Table XIX shows EOM-DEA-CCSD excitation energies computed using neutral reference orbitals. In this calculation, excitation energies are overestimated relative to exFCI. These results indicate the effect of the reference orbitals on the computed excitation energies.

Table XX and XXI compare the state and transition properties computed with EOM-EE-CCSD and EOM-DEA-CCSD (aug-cc-pVQZ) for ammonia and water, respectively. All properties are very close, confirming that EOM-DEA-CCSD and EOM-EE-CCSD yield wave function of broadly similar quality for singly excited states, despite using dicationic orbitals.

\section{J. Conical intersection in $\mathrm{HeH}_{2}$}

It is well established that EOM-EE-CC methods have defects ${ }^{44,75-79}$ close to conical intersections between excited states of the same symmetry. Yet, because the excited states are described on the same footing, one can obtain a physically correct description by appropriately modifying the EOM-CC method. ${ }^{78,80}$ Ground state intersections present a more difficult challenge: the intersecting states are described on an unequal footing and the $\mathrm{CC}$ ground-state wave function does not 
TABLE XVIII. Ammonia. Vertical excitation energies (eV) for the four lowest singlets and the lowest triplet states with different methods; aug-cc-pVQZ basis. Geometry from Ref. 59.

\begin{tabular}{lcccccc}
\hline \hline State & EE-CC $^{a}$ & EE-CCSDT $^{a}$ & exFCI $^{a}$ & EE-CCSD $^{b}$ & DEA-CCSD $^{b}$ & Exp. $^{c}$ \\
\hline \hline${ }^{1} \mathrm{~A}_{2}(\mathrm{n} \rightarrow 3 \mathrm{~s})$ & 6.61 & 6.61 & 6.64 & 6.67 & 6.14 & 6.39 \\
${ }^{1} \mathrm{E}(\mathrm{n} \rightarrow 3 \mathrm{p})$ & 8.18 & 8.17 & 8.22 & 8.21 & 7.66 & 7.93 \\
${ }^{1} \mathrm{~A}_{1}(\mathrm{n} \rightarrow 3 \mathrm{p})$ & 9.11 & 9.10 & 9.14 & 9.15 & 8.68 & 8.26 \\
${ }^{1} \mathrm{~A}_{2}(\mathrm{n} \rightarrow 4 \mathrm{~s})$ & 9.96 & 9.77 & 9.77 & 9.81 & 9.27 & \\
${ }^{3} \mathrm{~A}_{2}(\mathrm{n} \rightarrow 3 \mathrm{~s})$ & 6.31 & 6.33 & 6.35 & 6.37 & 5.85 & $6.02^{d}$ \\
\hline \hline
\end{tabular}

${ }^{a}$ Ref. 59. ${ }^{b}$ This work. ${ }^{c}$ Electron impact experiment from Ref. $72 .{ }^{d}$ Ref. 73.

TABLE XIX. Water. Vertical excitation energies (eV) for the 3 lowest singlet and 3 lowest triplet states computed with different methods; aug-cc-pVQZ basis. Geometry from Ref. 59.

\begin{tabular}{lccccccc}
\hline \hline State & EE-CC $^{a}$ & EE-CCSDT $^{a}$ & exFCI $^{a}$ & EE-CCSD $^{b}$ & DEA-CCSD/+2 $^{b}$ & DEA-CCSD/0 $^{b}$ & Exp. $^{c}$ \\
\hline${ }^{1} \mathrm{~B}_{1}\left(\mathrm{n}_{y} \rightarrow 3 \mathrm{~s}\right)$ & 7.65 & 7.64 & 7.68 & 7.68 & 6.97 & 7.92 & 7.41 \\
${ }^{1} \mathrm{~A}_{2}\left(\mathrm{n}_{z} \rightarrow 3 \mathrm{p}\right)$ & 9.43 & 9.41 & 9.46 & 9.44 & 8.72 & 9.70 & 9.20 \\
${ }^{1} \mathrm{~A}_{1}\left(\mathrm{n}_{z} \rightarrow 3 \mathrm{~s}\right)$ & 10.00 & 9.98 & 10.02 & 10.02 & 10.49 & 11.28 & 9.67 \\
${ }^{3} \mathrm{~B}_{1}\left(\mathrm{n}_{y} \rightarrow 3 \mathrm{~s}\right)$ & 7.28 & 7.26 & 7.30 & 7.29 & 6.62 & 7.53 & 7.20 \\
${ }^{3} \mathrm{~A}_{2}\left(\mathrm{n}_{z} \rightarrow 3 \mathrm{p}\right)$ & 9.26 & 9.25 & 9.28 & 9.27 & 8.57 & 9.53 & 8.90 \\
${ }^{3} \mathrm{~A}_{1}\left(\mathrm{n}_{z} \rightarrow 3 \mathrm{~s}\right)$ & 9.56 & 9.54 & 9.58 & 9.55 & 9.74 & 10.66 & 9.46 \\
\hline \hline
\end{tabular}

${ }^{a}$ Ref. 59. ${ }^{b}$ This work. ${ }^{c}$ Energy loss experiment from ref. 74.

TABLE XX. Ammonia. One-particle state and transition properties computed with EOM-EE-CCSD and EOM-DEA-CCSD; aug-ccpVQZ basis set.

\begin{tabular}{lccccc}
\hline \hline State & $\mathrm{n}_{u, n l}$ & $f_{l}$ & $\|\gamma\|$ & $Z_{H E}$ & e-h sep \\
\hline \hline EOM-EE-CCSD & & & & & \\
\hline${ }^{1} \mathrm{~A}_{2}(\mathrm{n} \rightarrow 3 \mathrm{~s})$ & 2.00 & 0.08 & 0.91 & 1.02 & 3.02 \\
${ }^{1} \mathrm{E}(\mathrm{n} \rightarrow 3 \mathrm{p})$ & 2.00 & 0.00 & 0.92 & 1.01 & 3.79 \\
${ }^{1} \mathrm{~A}_{1}(\mathrm{n} \rightarrow 3 \mathrm{p})$ & 1.91 & 0.00 & 0.91 & 1.19 & 3.46 \\
${ }^{1} \mathrm{~A}_{2}(\mathrm{n} \rightarrow 4 \mathrm{~s})$ & 2.00 & 0.00 & 0.91 & 1.01 & 4.57 \\
${ }^{3} \mathrm{~A}_{2}(\mathrm{n} \rightarrow 3 \mathrm{~s})$ & 2.00 & 0.00 & 0.92 & 1.05 & 2.86 \\
\hline${ }^{\mathrm{EOM}-\mathrm{DEA}-C C S D}$ & & & & & \\
${ }^{1} \mathrm{~A}_{2}(\mathrm{n} \rightarrow 3 \mathrm{~s})$ & 1.99 & 0.10 & 0.92 & 1.01 & 3.04 \\
${ }^{1} \mathrm{E}(\mathrm{n} \rightarrow 3 \mathrm{p})$ & 2.00 & 0.00 & 0.92 & 1.00 & 3.80 \\
${ }^{1} \mathrm{~A}_{1}(\mathrm{n} \rightarrow 3 \mathrm{p})$ & 1.82 & 0.00 & 0.91 & 1.07 & 3.52 \\
${ }^{1} \mathrm{~A}_{2}(\mathrm{n} \rightarrow 4 \mathrm{~s})$ & 2.00 & 0.00 & 0.92 & 1.00 & 4.57 \\
${ }^{3} \mathrm{~A}_{2}(\mathrm{n} \rightarrow 3 \mathrm{~s})$ & 2.00 & 0.00 & 0.92 & 1.03 & 2.89 \\
\hline \hline \multicolumn{5}{c}{$a$ e-h sep = electron hole separation in $\AA}$.
\end{tabular}

adequately capture the required multi-configurational character. These drawbacks can be removed by changing the reference wave function, as is done in EOM-SF, EOM-DIP, and EOM-DEA. In these cases both states are associated with nonzero eigenvalues of the Jacobian and the intersections are expected to resemble those between excited states in EOM-EECC.

The $\mathrm{HeH}_{2}$ system provides a simple test case for describing near-degeneracies between the ground state and first excited state. The states can be characterized by inspecting the natural orbitals and their occupations (see Fig. 12). At short bond
TABLE XXI. Water. One-particle state and transition properties computed with EOM-EE-CCSD and EOM-DEA-CCSD; aug-ccpVQZ basis set.

\begin{tabular}{|c|c|c|c|c|c|}
\hline State & $\mathrm{n}_{u, n l}$ & $f_{l}$ & $|\gamma| \mid$ & $Z_{H E}$ & e-h sep ${ }^{a}$ \\
\hline \multicolumn{6}{|c|}{ EOM-EE-CCSD } \\
\hline${ }^{1} \mathrm{~B}_{1}\left(\mathrm{n}_{y} \rightarrow 3 \mathrm{~s}\right)$ & 2.00 & 0.05 & 0.92 & 1.01 & 2.60 \\
\hline${ }^{1} \mathrm{~A}_{2}\left(\mathrm{n}_{z} \rightarrow 3 \mathrm{p}\right)$ & 2.00 & 0.00 & 0.92 & 1.01 & 3.22 \\
\hline${ }^{1} \mathrm{~A}_{1}\left(\mathrm{n}_{z} \rightarrow 3 \mathrm{~s}\right)$ & 2.00 & 0.10 & 0.92 & 1.55 & 2.67 \\
\hline${ }^{3} \mathrm{~B}_{1}\left(\mathrm{n}_{y} \rightarrow 3 \mathrm{~s}\right)$ & 2.00 & 0.00 & 0.92 & 1.04 & 2.45 \\
\hline${ }^{3} \mathrm{~A}_{2}\left(\mathrm{n}_{z} \rightarrow 3 \mathrm{p}\right)$ & 2.00 & 0.00 & 0.92 & 1.02 & 3.06 \\
\hline${ }^{3} \mathrm{~A}_{1}\left(\mathrm{n}_{z} \rightarrow 3 \mathrm{~s}\right)$ & 2.00 & 0.00 & 0.92 & 1.12 & 2.49 \\
\hline \multicolumn{6}{|c|}{ EOM-DEA-CCSD } \\
\hline${ }^{1} \mathrm{~B}_{1}\left(\mathrm{n}_{y} \rightarrow 3 \mathrm{~s}\right)$ & 2.00 & 0.06 & 0.92 & 1.01 & 2.63 \\
\hline${ }^{1} \mathrm{~A}_{2}\left(\mathrm{n}_{z} \rightarrow 3 \mathrm{p}\right)$ & 2.00 & 0.00 & 0.92 & 1.00 & 3.23 \\
\hline${ }^{1} \mathrm{~A}_{1}\left(\mathrm{n}_{z} \rightarrow 3 \mathrm{~s}\right)$ & 1.82 & 0.00 & 0.92 & 1.12 & 3.00 \\
\hline${ }^{3} \mathrm{~B}_{1}\left(\mathrm{n}_{y} \rightarrow 3 \mathrm{~s}\right)$ & 2.00 & 0.00 & 0.93 & 1.02 & 2.48 \\
\hline${ }^{3} \mathrm{~A}_{2}\left(\mathrm{n}_{z} \rightarrow 3 \mathrm{p}\right)$ & 2.00 & 0.00 & 0.92 & 1.01 & 3.09 \\
\hline${ }^{3} \mathrm{~A}_{1}\left(\mathrm{n}_{z} \rightarrow 3 \mathrm{~s}\right)$ & 2.00 & 0.00 & 0.94 & 1.07 & 2.78 \\
\hline
\end{tabular}

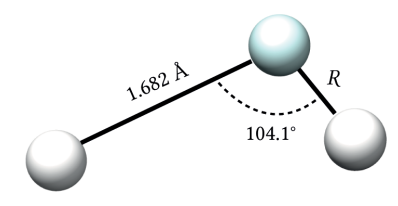

FIG. 11. $\mathrm{HeH}_{2}$ molecule. One He-H bond length is varied $(R)$.

lengths, the ground state has two electrons in the $1 a^{\prime}$ and $2 a^{\prime}$ 
orbitals. At the long bond lengths, one electron has moved from $2 a^{\prime}$ to $3 a^{\prime}$. The situation is reversed for the first excited state.

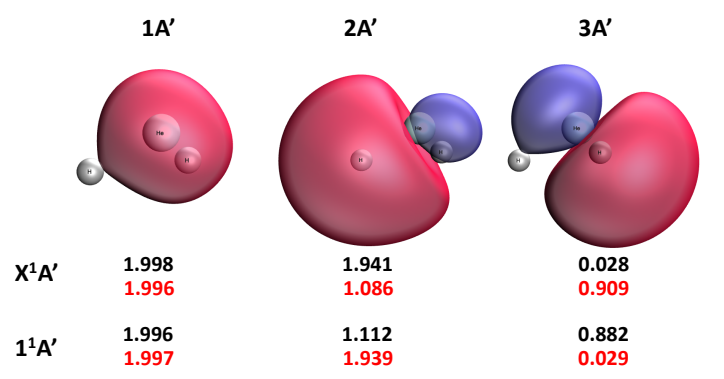

FIG. 12. Natural orbitals and their occupation numbers for ground and first excited state. Black color corresponds to geometry at $\mathrm{He}-\mathrm{H}$ $=0.6 \AA$. Red color corresponds to geometry at $\mathrm{He}-\mathrm{H}=0.8 \AA$.

We consider a set of $\mathrm{C}_{s}$ geometries where one of the $\mathrm{He}-\mathrm{H}$ bond lengths is varied (see Fig. 11). Figure 13 shows potential energy curves obtained using EOM-EE-CCSD, EOMDEA-CCSD, and FCI. The EOM-EE-CCSD and EOM-DEACCSD curves are similar but shifted relative to FCI. However, upon closer inspection, we see that EOM-EE-CCSD has defective points and a region where we are not able to converge the equations because EOM-CC roots become complex (see the right panel of Fig. 13). In this particular scan, EOMDEA-CCSD gives an avoided crossing. Closer to the intersection, we expect to see complex pairs and defects in EOMDEA-CCSD - just like for excited states in EOM-EE-CCSD. However, EOM-DEA-CCSD should give a generally more accurate description of the intersecting states. Furthermore, defects encountered with EOM-DEA-CCSD can be removed by using the similarity constrained CC approach. ${ }^{78,80}$
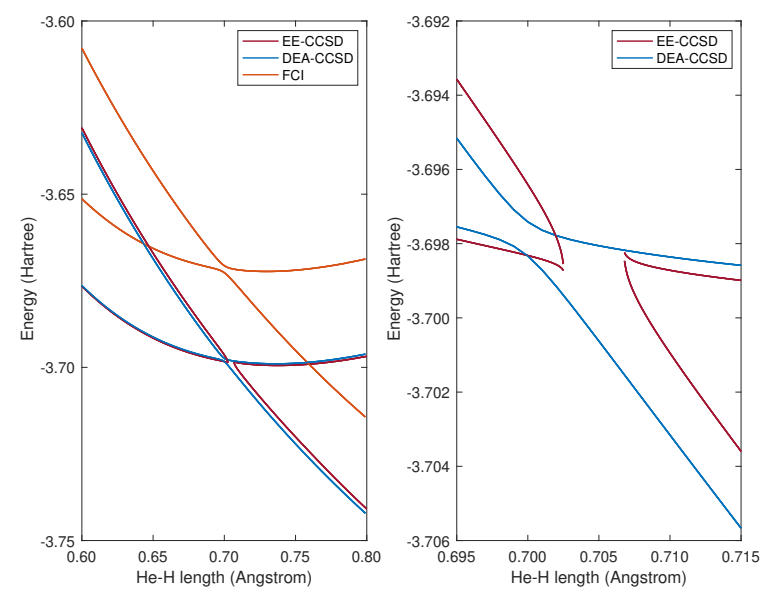

FIG. 13. Conical intersection between $X^{1} \mathrm{~A}^{\prime}$ and $1^{1} \mathrm{~A}^{\prime}$ computed using EOM-DEA-CCSD, EOM-EE-CCSD, and full configuration interaction (FCI) methods with the aug-cc-pVDZ basis set. The left pane shows all three limits for the full range of bond lengths ( 0.6 to $0.8 \AA$ ). The right pane zooms in on the near-degeneracy for EOMDEA-CCSD and EOM-EE-CCSD.

\section{CONCLUSIONS}

In this contribution, we have documented a productionlevel implementation of EOM-DEA-CCSD, including calculation of state and transition properties. This ansatz is suitable for treating electronic structure patterns that can be described as two-electrons-in-many orbitals. Hence, it extends the scope of applicability of EOM-CC methods, including the domain traditionally described as multi-reference ${ }^{22}$. Following the pioneering work of Bartlett and coworkers ${ }^{16,17,21}$, here we illustrate the advantages and limitations of EOM-DEA-CCSD relative to other EOM-CCSD methods (EOM-EE-CCSD and EOM-SF-CCSD) by considering a diverse set of examples comprising well-behaved singly excited states, doubly excited states, diradicals, bond-breaking, and conical intersections. The results can be summarized as follows:

1. Excited states in closed-shell molecules, which can be qualitatively characterized as excitations from the HOMO, are described with similar accuracy as in EOM-EE-CCSD; the performance for excited states derived by excitations from lower occupied orbitals is poor, as the method is clearly not designed for such applications.

2. Diradical states are described as accurately as by EOMSF-CCSD, but within spin-adapted framework. The benefits are no spin-contamination and faster calculation. Furthermore, EOM-DEA-CCSD allows access to higher excited states, beyond primary SF manifold.

3. EOM-DEA-CCSD can describe states with significant or even pure doubly excited character.

4. EOM-DEA-CCSD describes PES along bond breaking reasonably well, as illustrated in ethylene torsion example.

5. EOM-DEA-CCSD can also treat some problems involving conical intersections, where standard EOMEE-CCSD is inapplicable.

6. Orbital relaxation is somewhat of a problem. In most cases, using default dicationic reference orbitals yields robust performance, but sometimes the charge of the reference needs to be tweaked.

The results show that EOM-DEA-CCSD represents a useful addition to EOM-CC family of methods. We hope that this contribution provides a useful guide for choosing the most appropriate EOM-CC method to tackle specific applications.

\section{ACKNOWLEDGMENTS}

This work is supported by the Department of Energy through the DE-SC0018910 grant (A.I.K.). J.F.S. acknowledges support from the National Science Foundation (CHE1748821). H.K. and E.F.K. acknowledge funding from the Marie Skłodowska-Curie European Training Network COSINE (Computational Spectroscopy in Natural Sciences and 
Engineering) Grant Agreement No. 765739, and the Research Council of Norway through FRINATEK Projects No. 263110 and No. 275506.

\section{CONFLICTS OF INTEREST}

A.I.K. is the president and a part-owner of Q-Chem, Inc. 


\section{APPENDIX A}

The left and right EOM $\sigma$-vectors are defined as:

$$
\begin{aligned}
& \sigma_{1}=\left(\left[\bar{H}_{S S}-E_{c c}\right] R_{1}\right)_{1}+\left(\bar{H}_{S D} R_{2}\right)_{1}, \\
& \sigma_{2}=\left(\bar{H}_{D S} R_{1}\right)_{2}+\left(\left[\bar{H}_{D D}-E_{c c}\right] R_{2}\right)_{2}, \\
& \tilde{\sigma}_{1}=\left(L_{1}\left[\bar{H}_{S S}-E_{c c}\right]\right)_{1}+\left(L_{2} \bar{H}_{D S}\right)_{1}, \\
& \tilde{\sigma}_{2}=\left(L_{1} \bar{H}_{S D}\right)_{2}+\left(L_{2}\left[\bar{H}_{D D}-E_{c c}\right]\right)_{2} .
\end{aligned}
$$

TABLE XXII. Programmable expressions for the right $(\sigma)$ and left $(\tilde{\sigma})$ vectors in EOM-DEA-CCSD.

$$
\begin{gathered}
\sigma^{a b}=P(a b)\left(\sum_{c} F_{b c} r^{a c}-\frac{1}{2} \sum_{i c d} I_{i b c d}^{7} r_{i}^{a c d}\right) \\
+\frac{1}{2} \sum_{c d} r^{c d} I_{a b c d}^{5}+\sum_{i c} F_{i c} r_{i}^{a b c} \\
\sigma_{i}^{a b c}=\frac{1}{2} P(a b c)\left(\sum_{d} F_{c d} r_{i}^{a b d}-\sum_{j d} I_{i d j c}^{1} r_{j}^{a b d}+\frac{1}{2} \sum_{d e} I_{a b d e}^{5} r_{i}^{c d e}\right. \\
\left.-\sum_{j} H_{j c}^{6} t_{i j}^{a b}-\sum_{d} I_{i d a b}^{3} r_{c d}\right)-\sum_{j} F_{i j} r_{j}^{a b c} \\
\tilde{\sigma}^{a b}=P(a b)\left(\sum_{c} l^{a c} F_{a c}-\frac{1}{2} \sum_{i c d} l_{i}^{a c d} I_{i b c d}^{3}\right)-\sum_{i c} H_{i c}^{7} I_{i c a b}^{7} \\
+\frac{1}{2} \sum_{c d} l^{c d} I_{c d a b}^{5} \\
\left.+\frac{1}{2} \sum_{d e} l_{i}^{a d e} I_{d e b c}^{5}-\sum_{k} H_{k a}^{7}\langle i k \| b c\rangle+l^{a b} F_{i c}-\sum_{d} l^{c d} I_{i d a b}^{7}\right) \\
-\sum_{j} l_{j}^{a b c} F_{j i}
\end{gathered}
$$

TABLE XXIII. Intermediates used in EOM-DEA-CCSD $\sigma$-vectors.

$$
\begin{aligned}
& F_{i a}=f_{i a}+\sum_{j b} t_{j}^{b}\langle i j \| a b\rangle \\
& F_{i j}=f_{i j}+\sum_{a} t_{i}^{a} f_{j a}+\sum_{k a} t_{k}^{a}\langle j k \| i a\rangle+\sum_{k a b} t_{i}^{a} t_{k}^{b}\langle j k \| a b\rangle+ \\
& \frac{1}{2} \sum_{k b c} t_{i k}^{b c}\langle j k \| b c\rangle \\
& F_{a b}=f_{a b}-\sum_{i} t_{i}^{a} f_{i b}-\sum_{i c} t_{i}^{c}\langle i a \| b c\rangle+\sum_{i j c} t_{i}^{c} t_{j}^{a}\langle i j|| b c\rangle- \\
& \frac{1}{2} \sum_{j k c} t_{j k}^{a c}\langle j k|| b c\rangle \\
& I_{i a j b}^{1}=\langle i a|| j b\rangle-\sum_{k} t_{k}^{b}\langle j k \| i a\rangle-\sum_{c} t_{i}^{c}\langle j b \| a c\rangle+ \\
& \sum_{k c} t_{i}^{c} t_{k}^{b}\langle j k \| a c\rangle-\sum_{k c} t_{i k}^{b c}\langle j k \| a c\rangle \\
& I_{i c a b}^{3}=-\langle i c|| a b\rangle+2 \sum_{d} t_{i}^{d} I_{b c a d}^{5}+ \\
& \sum_{j}\left(t_{j}^{b}\left(\langle i a \| j c\rangle-\sum_{k d} t_{i k}^{c d}\langle k j|| a d\rangle\right)-t_{j}^{c}(\langle i a \| j b\rangle-\right. \\
& \left.\left.\sum_{k d} t_{i k}^{b d}\langle j k \| a d\rangle\right)\right)- \\
& \sum_{j k} t_{j}^{b} t_{k}^{c}\langle j k \| i a\rangle-\sum_{j k d} t_{j}^{d} t_{i k}^{b c}\langle j k \| \mid a d\rangle+ \\
& \sum_{k} t_{i k}^{a b} f_{k c}-\frac{1}{2} \sum_{k l} t_{k l}^{a b}\langle i c|| k l\rangle+ \\
& \sum_{k d}\left(t_{i k}^{a d}\langle k b \| c d\rangle-t_{i k}^{b d}\langle k a \| c d\rangle\right) \\
& I_{a b c d}^{5}=\langle a b \| c d\rangle+\frac{1}{2} \sum_{i j} \tilde{t}_{i j}^{a b}\langle i j \| c d\rangle-P(a b) \sum_{i} t_{i}^{a}\langle i b \| c d\rangle \\
& I_{i a b c}^{7}=\langle i a \| b c\rangle-\sum_{j} t_{j}^{a}\langle i j \| b c\rangle \\
& \tilde{t}_{i j}^{a b}=t_{i j}^{a b}+\frac{1}{2} P(i j) P(a b) t_{i}^{a} t_{j}^{b} \\
& H_{i a}^{6}=\frac{1}{2} \sum_{b c} r^{b c} I_{i a b c}^{7}+\frac{1}{2} \sum_{j b c} r_{j}^{a b c}\langle j i \| b c\rangle \\
& H_{i a}^{7}=\frac{1}{2} \sum_{j b c} l_{j}^{a b c} t_{j i}^{b c}
\end{aligned}
$$


TABLE XXIV. Programmable expressions for EOM-DEA-CCSD density matrices.

\begin{tabular}{c}
\hline \hline$\gamma_{i j}=\tilde{\gamma}_{i j}+\delta_{i j}$ \\
$\tilde{\gamma}_{i j}=-\tilde{l}_{i j}-\sum_{a} Y_{j a}^{1} t_{i}^{a}$ \\
$\gamma_{a i}=Y_{i a}^{1}$ \\
$\gamma_{a b}=\tilde{l}^{a b}+Y_{i a}^{1} t_{i}^{b}$ \\
$\gamma_{i a}=t_{i}^{a}+\sum_{j b} Y_{j b}^{1}\left(t_{i j}^{a b}-t_{j}^{a} t_{i}^{b}\right)+\frac{1}{2} \sum_{b c} l^{b c} r_{i}^{a b c}$ \\
$-\sum_{k} \tilde{l}_{i k} t_{k}^{a}-\sum_{b} \tilde{l}^{a b} t_{i}^{b}+\sum_{b} Y_{i b}^{2} r^{a b}$ \\
$\tilde{l}_{i j}=\frac{1}{6} \sum_{a b c} l_{j}^{a b c} r_{i}^{a b c}$ \\
$\tilde{l}^{a b}=\sum_{c} l^{a c} r^{b c}+\frac{1}{2} \sum_{i c d} l_{i}^{a c d} r_{i}^{b c d}$ \\
$Y_{i a}^{1}=\frac{1}{2} \sum_{b c} l_{i}^{a b c} r^{b c}$ \\
$Y_{i a}^{2}=\frac{1}{2} \sum_{j b c} l_{j}^{a b c} t_{i j}^{b c}$
\end{tabular}


${ }^{1}$ R. J. Bartlett, How and why coupled-cluster theory became the preeminent method in $a b$ initio quantum chemistry, In C. Dykstra, G. Frenking, and G. Scuseria, editors, Theory and Applications of computational chemistry, Elsevier, (2005).

${ }^{2}$ R. J. Bartlett, The coupled-cluster revolution, Mol. Phys., 108, 2905-2920 (2010).

${ }^{3}$ G. D. Purvis and R. J. Bartlett, A full coupled-cluster singles and doubles model: The inclusion of disconnected triples, J. Chem. Phys., 76, 19101918 (1982)

${ }^{4}$ K. Raghavachari, G.W . Trucks, J. A. Pople, and M. Head-Gordon, A fifthorder perturbation comparison of electron correlation theories, Chem. Phys. Lett., 157, 479-483 (1989).

${ }^{5} \mathrm{~K}$. Emrich, An extension of the coupled-cluster formalism to excited states (I), Nucl. Phys., A351, 379-396 (1981).

${ }^{6} \mathrm{H}$. Sekino and R. J. Bartlett, A linear response, coupled-cluster theory for excitation energy, Int. J. Quant. Chem., 26, 255-265 (1984).

${ }^{7} \mathrm{H}$. Koch and P. Jørgensen, Coupled cluster response functions, J. Chem. Phys., 93(5), 3333-3344 (1990).

${ }^{8}$ H. Koch, H.J.Aa. Jensen, P. Jørgensen, and T. Helgaker, Excitation energies from the coupled clusters singles and doubles linear response functions (CCSDLR). Applications to $\mathrm{Be}, \mathrm{CH}^{+}, \mathrm{CO}$, and $\mathrm{H}_{2} \mathrm{O}$, J. Chem. Phys., 93(5), 3345-3350 (1990).

${ }^{9}$ J. F. Stanton and R. J. Bartlett, The equation of motion coupled-cluster method. A systematic biorthogonal approach to molecular excitation energies, transition probabilities, and excited state properties, J. Chem. Phys., 98, 7029-7039 (1993).

${ }^{10} \mathrm{M}$. Nooijen and R. J. Bartlett, Description of core-excitation spectra by the open-shell electron-attachment equation-of-motion coupled cluster method, J. Chem. Phys., 102, 6735-6756 (1995).

${ }^{11}$ S. V. Levchenko and A. I. Krylov, Equation-of-motion spin-flip coupledcluster model with single and double substitutions: Theory and application to cyclobutadiene, J. Chem. Phys., 120(1), 175-185 (2004).

${ }^{12}$ A. I. Krylov, Equation-of-motion coupled-cluster methods for open-shell and electronically excited species: The hitchhiker's guide to Fock space, Annu. Rev. Phys. Chem., 59, 433-462 (2008).

${ }^{13} \mathrm{~K}$. Sneskov and O. Christiansen, Excited state coupled cluster methods, WIREs: Comput. Mol. Sci., 2, 566-584 (2012).

${ }^{14}$ R. J. Bartlett, Coupled-cluster theory and its equation-of-motion extensions, WIREs: Comput. Mol. Sci., 2(1), 126-138 (2012).

${ }^{15}$ A. I. Krylov, The quantum chemistry of open-shell species, In A. L. Parrill and K. B. Lipkowitz, editors, Reviews in Comp. Chem., volume 30, pages 151-224, J. Wiley \& Sons, (2017).

${ }^{16} \mathrm{M}$. Nooijen and R. J. Bartlett, Similarity transformed equation-of-motion coupled-cluster theory: Details, examples, and comparisons, J. Chem. Phys., 107, 6812-6830 (1997).

${ }^{17}$ M. Musiaz, S. A. Kucharski, and R. J. Bartlett, Multireference double electron attached coupled cluster method with full inclusion of the connected triple excitations: MR-DA-CCSDT, J. Chem. Theory Comput., 7, 30883096 (2011).

${ }^{18}$ M. Tobita, S.A. Perera, M. Musial, R.J. Bartlett, M. Nooijen, and J.S. Lee, Critical comparison of single-reference and multireference coupledcluster methods: Geometry, harmonic frequencies, and excitation energies of $\mathrm{N}_{2} \mathrm{O}_{2}$, J. Chem. Phys., 119, 10713-10723 (2003).

${ }^{19}$ M. K. Nayak and R. K. Chaudhuri, Relativistic coupled cluster method, Eur. Phys. J. D, 37, 171-176 (2006).

${ }^{20}$ D. Bokhan, D. N. Trubnikov, A. Perera, and R. J. Bartlett, Explicitlycorrelated double ionization potentials and double electron attachment equation-of-motion coupled cluster methods, Chem. Phys. Lett., 692, 191195 (2018).

${ }^{21}$ A. Perera, R. W. Molt, V. F. Lotrich, and R. J. Bartlett, Singlet-triplet separations of di-radicals treated by the DEA/DIP-EOM-CCSD methods, In C. J. Cramer and D. G. Truhlar, editors, Isaiah Shavitt, volume 9 of $A$ memorial festschrift from theoretical chemistry accounts, pages 153-165, Springer, (2016).

${ }^{22} \mathrm{R}$. J. Bartlett, To multireference or not to multireference: That is the question?, Int. J. Mol. Sci., 3, 579-603 (2002).

${ }^{23}$ D. Casanova and A. I. Krylov, Spin-flip methods in quantum chemistry, Phys. Chem. Chem. Phys., 22, 4326-4342 (2020).

${ }^{24} \mathrm{M}$. Nooijen and R. J. Bartlett, Equation of motion coupled cluster method for electron attachment, J. Chem. Phys., 102, 3629-3647 (1995).
${ }^{25} \mathrm{M}$. Guo, Z. Wang, and F. Wang, Equation-of-motion coupled-cluster theory for double electron attachment with spin-orbit coupling, J. Chem. Phys., 153(21), 214118 (2020).

${ }^{26}$ A. I. Krylov, From orbitals to observables and back, J. Chem. Phys., 153, 080901 (2020).

${ }^{27}$ S. Mewes, F. Plasser, A. I. Krylov, and A. Dreuw, Benchmarking excitedstate calculations using exciton properties, J. Chem. Theory Comput., 14, 710-725 (2018).

${ }^{28}$ P.-O. Löwdin, Quantum theory of many-particle systems. I. Physical interpretations by means of density matrices, natural spin-orbitals, and convergence problems in the method of configurational interaction, Phys. Rev., 97, 1474-1489 (1955).

${ }^{29} \mathrm{~K}$. Takatsuka, T. Fueno, and K. Yamaguchi, Distribution of odd electrons in ground-state molecules, Theor. Chim. Acta, 48, 175-183 (1978).

${ }^{30} \mathrm{M}$. Head-Gordon, Characterizing unpaired electrons from the one-particle density matrix, Chem. Phys. Lett., 372, 508-511 (2003).

${ }^{31}$ A. V. Luzanov, Effectively unpaired electrons for singlet states: from diatomics to graphene nanoclusters, In J. Leszczynski and M. Shukla, editors, Practical Aspects of Computational Chemistry IV, chapter 6, pages 151-206, Springer-Verlag, New York, (2016).

${ }^{32}$ A. V. Luzanov, A. A. Sukhorukov, and V. E. Umanskii, Application of transition density matrix for analysis of excited states, Theor. Exp. Chem., 10, 354-361 (1976), Russian original: Teor. Eksp. Khim., 10, 456 (1974).

${ }^{33}$ A. V. Luzanov and V. F. Pedash, Interpretation of excited states using charge-transfer number, Theor. Exp. Chem., 15, 338-341 (1979).

${ }^{34}$ A. V. Luzanov and O. A. Zhikol, Excited state structural analysis: TDDFT and related models, In J. Leszczynski and M.K. Shukla, editors, Practical aspects of computational chemistry I: An overview of the last two decades and current trends, pages 415-449, Springer, (2012).

${ }^{35}$ F. Plasser, M. Wormit, and A. Dreuw, New tools for the systematic analysis and visualization of electronic excitations. I. Formalism, J. Chem. Phys., 141, 024106-13 (2014).

${ }^{36}$ F. Plasser, S. A. Bäppler, M. Wormit, and A. Dreuw, New tools for the systematic analysis and visualization of electronic excitations. II. Applications, J. Chem. Phys., 141, 024107-12 (2014).

${ }^{37}$ F. Plasser, Entanglement entropy of electronic excitations, J. Chem. Phys., 144, 194107 (2016).

${ }^{38} \mathrm{P}$. Kimber and F. Plasser, Toward an understanding of electronic excitation energies beyond the molecular orbital picture, Phys. Chem. Chem. Phys., 22, 6058-6080 (2020).

${ }^{39}$ L. V. Slipchenko and A. I. Krylov, Singlet-triplet gaps in diradicals by the spin-flip approach: A benchmark study, J. Chem. Phys., 117, 4694-4708 (2002).

${ }^{40}$ L. V. Slipchenko and A. I. Krylov, Electronic structure of the trimethylenemethane diradical in its ground and electronically excited states: Bonding, equilibrium structures and vibrational frequencies, J. Chem. Phys., 118, 6874-6883 (2003).

${ }^{41}$ P. U. Manohar and A. I. Krylov, A non-iterative perturbative triples correction for the spin-flipping and spin-conserving equation-of-motion coupledcluster methods with single and double substitutions, J. Chem. Phys., 129, 194105 (2008).

${ }^{42}$ M. V. Ivanov, S. Gulania, and A. I. Krylov, Two cycling centers in one molecule: Communication by through-bond interactions and entanglement of the unpaired electrons, J. Phys. Chem. Lett., 11(4), 1297-1304 (2020), in press.

${ }^{43}$ M. Ivanov, A. I. Krylov, and S. Zilberg, Long-range N-N bonding by Rydberg electrons, J. Phys. Chem. Lett., 11, 2284-2290 (2020).

${ }^{44} \mathrm{~S}$. Gozem, A. I. Krylov, and M. Olivucci, Conical intersection and potential energy surface features of a model retinal chromophore: Comparison of eom-cc and multireference methods, J. Chem. Theory Comput., 9, 284292 (2013).

${ }^{45}$ S. Gozem, F. Melaccio, R. Lindh, A. I. Krylov, A. A. Granovsky, C. Angeli, and M. Olivucci, Mapping the excited state potential energy surface of a retinal chromophore model with multireference and equation-of-motion coupled-cluster methods, J. Chem. Theory Comput., 9, 4495-4506 (2013). ${ }^{46}$ S. Gozem, F. Melaccio, A. Valentini, M. Filatov, M. Huix-Rotllant, N. Ferre, L. M. Frutos, C. Angeli, A. I. Krylov, A. A. Granovsky, R. Lindh, and M. Olivucci, Shape of multireference, EOM-CC, and DFT potential energy surfaces at a conical intersection, J. Chem. Theory Comput., 10, 3074-3084 (2014). 
${ }^{47}$ A. I. Krylov and P. M. W. Gill, Q-Chem: An engine for innovation, WIREs: Comput. Mol. Sci., 3, 317-326 (2013).

${ }^{48}$ Y. Shao, Z. Gan, E. Epifanovsky, A.T.B. Gilbert, M. Wormit, J. Kussmann, A.W. Lange, A. Behn, J. Deng, X. Feng, D. Ghosh, M. Goldey, P.R. Horn, L.D. Jacobson, I. Kaliman, R.Z. Khaliullin, T. Kus, A. Landau, J. Liu, E.I. Proynov, Y.M. Rhee, R.M. Richard, M.A. Rohrdanz, R.P. Steele, E.J. Sundstrom, H.L. Woodcock III, P.M. Zimmerman, D. Zuev, B. Albrecht, E. Alguires, B. Austin, G.J.O. Beran, Y.A. Bernard, E. Berquist, K. Brandhorst, K.B. Bravaya, S.T. Brown, D. Casanova, C.-M. Chang, Y. Chen, S.H. Chien, K.D. Closser, D.L. Crittenden, M. Diedenhofen, R.A. DiStasio Jr., H. Do, A.D. Dutoi, R.G. Edgar, S. Fatehi, L. Fusti-Molnar, A. Ghysels, A. Golubeva-Zadorozhnaya, J. Gomes, M.W.D. Hanson-Heine, P.H.P. Harbach, A.W. Hauser, E.G. Hohenstein, Z.C. Holden, T.-C. Jagau, H. Ji, B. Kaduk, K. Khistyaev, J. Kim, J. Kim, R.A. King, P. Klunzinger, D. Kosenkov, T. Kowalczyk, C.M. Krauter, K.U. Laog, A. Laurent, K.V. Lawler, S.V. Levchenko, C.Y. Lin, F. Liu, E. Livshits, R.C. Lochan, A. Luenser, P. Manohar, S.F. Manzer, S.-P. Mao, N. Mardirossian, A.V. Marenich, S.A. Maurer, N.J. Mayhall, C.M. Oana, R. Olivares-Amaya, D.P. O'Neill, J.A. Parkhill, T.M. Perrine, R. Peverati, P.A. Pieniazek, A. Prociuk, D.R. Rehn, E. Rosta, N.J. Russ, N. Sergueev, S.M. Sharada, S. Sharmaa, D.W. Small, A. Sodt, T. Stein, D. Stuck, Y.-C. Su, A.J.W. Thom, T. Tsuchimochi, L. Vogt, O. Vydrov, T. Wang, M.A. Watson, J. Wenzel, A. White, C.F. Williams, V. Vanovschi, S. Yeganeh, S.R. Yost, Z.-Q. You, I.Y. Zhang, X. Zhang, Y. Zhou, B.R. Brooks, G.K.L. Chan, D.M. Chipman, C.J. Cramer, W.A. Goddard III, M.S. Gordon, W.J. Hehre, A. Klamt, H.F. Schaefer III, M.W. Schmidt, C.D. Sherrill, D.G. Truhlar, A. Warshel, X. Xu, A. AspuruGuzik, R. Baer, A.T. Bell, N.A. Besley, J.-D. Chai, A. Dreuw, B.D. Dunietz, T.R. Furlani, S.R. Gwaltney, C.-P. Hsu, Y. Jung, J. Kong, D.S. Lambrecht, W.Z. Liang, C. Ochsenfeld, V.A. Rassolov, L.V. Slipchenko, J.E. Subotnik, T. Van Voorhis, J.M. Herbert, A.I. Krylov, P.M.W. Gill, and M. Head-Gordon, Advances in molecular quantum chemistry contained in the Q-Chem 4 program package, Mol. Phys., 113, 184-215 (2015).

${ }^{49} \mathrm{R}$. S. Mulliken, Report on notation for the spectra of polyatomic molecules, J. Chem. Phys., 23, 1997-2011 (1955).

${ }^{50}$ D. A. Matthews, L. Cheng, M. E. Harding, F. Lipparini, S. Stopkowicz, T-C. Jagau, P. G. Szalay, J. Gauss, and J. F. Stanton, Coupled-cluster techniques for computational chemistry: The cfour program package, J. Chem. Phys., 152, 214108 (2020)

${ }^{51}$ S. D. Folkestad, E. F. Kjønstad, R. H. Myhre, J. H. Andersen, A. Balbi, S. Coriani, T. Giovannini, L. Goletto, T. S. Haugland, A. Hutcheson, I-M. Høyvik, T. Moitra, A. C. Paul, M. Scavino, A. S. Skeidsvoll, ̊. H. Tveten, and H. Koch, et 1.0: An open source electronic structure program with emphasis on coupled cluster and multilevel methods, J. Chem. Phys., 152, 184103 (2020).

${ }^{52}$ A. C. Paul, R. H. Myhre, and H. Koch, New and efficient implementation of CC3, J. Chem. Theory Comput., $\mathbf{0}(0)$, null (0), in press.

${ }^{53}$ K. Aidas, C. Angeli, K. L. Bak, V. Bakken, R. Bast, L. Boman, O. Christiansen, R. Cimiraglia, S. Coriani, P. Dahle, E. K. Dalskov, U. Ekström, T. Enevoldsen, J. J. Eriksen, P. Ettenhuber, B. Fernández, L. Ferrighi, H. Fliegl, L. Frediani, K. Hald, A. Halkier, C. Hättig, H. Heiberg, T. Helgaker, A. C. Hennum, H. Hettema, E. Hjertenæs, S. Høst, I.-M. Høyvik, M. F. Iozzi, B. Jansik, H. J. Aa. Jensen, D. Jonsson, P. Jørgensen, J. Kauczor, S. Kirpekar, T. Kjærgaard, W. Klopper, S. Knecht, R. Kobayashi, H. Koch, J. Kongsted, A. Krapp, K. Kristensen, A. Ligabue, O. B. Lutnæs, J. I. Melo, K. V. Mikkelsen, R. H. Myhre, C. Neiss, C. B. Nielsen, P. Norman, J. Olsen, J. M. H. Olsen, A. Osted, M. J. Packer, F. Pawlowski, T. B. Pedersen, P. F. Provasi, S. Reine, Z. Rinkevicius, T. A. Ruden, K. Ruud, V. Rybkin, P. Salek, C. C. M. Samson, A. Sánchez de Merás, T. Saue, S. P. A. Sauer, B. Schimmelpfennig, K. Sneskov, A. H. Steindal, K. O. SylvesterHvid, P. R. Taylor, A. M. Teale, E. I. Tellgren, D. P. Tew, A. J. Thorvaldsen, L. Thøgersen, O. Vahtras, M. A. Watson, D. J. D. Wilson, M. Ziolkowski, and H. Agren, The Dalton quantum chemistry program system, WIREs: Comput. Mol. Sci., 4, 269-284 (2014).

${ }^{54}$ C.D. Sherrill, M.L. Leininger, T.J. Van Huis, and H.F. Schaefer III, Structures and vibrational frequencies in the full configuration interaction limit: Predictions for four electronic states of methylene using triple-zeta plus double polarization (TZ2P) basis, J. Chem. Phys., 108(3), 1040-1049 (1998).

${ }^{55}$ Y. Shao, M. Head-Gordon, and A. I. Krylov, The spin-flip approach within time-dependent density functional theory: Theory and applications to di- radicals, J. Chem. Phys., 118, 4807-4818 (2003).

${ }^{56} \mathrm{M}$. A. Watson and G. K. L. Chan, Excited states of butadiene to chemical accuracy: Reconciling theory and experiment, J. Chem. Theory Comput., 8, 4013-4018 (2012).

${ }^{57}$ A. I. Krylov, Size-consistent wave functions for bond-breaking: The equation-of-motion spin-flip model, Chem. Phys. Lett., 338, 375-384 (2001).

${ }^{58} \mathrm{~A}$. Kalemos and A. Mavridis, Electronic structure and bonding of ozone, J. Chem. Phys., 129, 054312 (2008)

${ }^{59}$ P. F. Loos, A. Scemama, A. Blondel, Y. Garniron, M. Caffarel, and D. Jacquemin, A mountaineering strategy to excited states: Highly accurate reference energies and benchmarks, J. Chem. Theory Comput., 14, 4360-4379 (2018).

${ }^{60}$ M. V. Ivanov, F. H. Bangerter, P. Wojcik, and A.I. Krylov, Towards ultracold organic chemistry: Prospects of laser cooling large organic molecules, J. Phys. Chem. Lett., 11, 6670-6676 (2020).

${ }^{61}$ V. Vanovschi, A. I. Krylov, and P. G. Wenthold, Structure, vibrational frequencies, ionization energies, and photoelectron spectrum of the parabenzyne radical anion, Theor. Chim. Acta, 120, 45-58 (2008).

${ }^{62}$ A. M. C. Cristian, Y. Shao, and A. I. Krylov, Bonding patterns in benzene triradicals from structural, spectroscopic, and thermochemical perspectives, J. Phys. Chem. A, 108, 6581-6588 (2004).

${ }^{63}$ Y. A. Bernard, Y. Shao, and A. I. Krylov, General formulation of spinflip time-dependent density functional theory using non-collinear kernels: Theory, implementation, and benchmarks, J. Chem. Phys., 136, 204103 (2012).

${ }^{64}$ A. Luxon, N. Orms, R. Kanters, A. I. Krylov, and C. Parish, An ab initio exploration of the Bergman cyclization, J. Phys. Chem. A, 122, 420-430 (2018).

${ }^{65}$ N. Orms, D. R. Rehn, A. Dreuw, and A. I. Krylov, Characterizing bonding patterns in diradicals and triradicals by density-based wave function analysis: A uniform approach, J. Chem. Theory Comput., 14, 638-648 (2018).

${ }^{66} \mathrm{P}$. G. Wenthold, J. Hu, and R. R. Squires, o-, m-, and p-benzyne negative ions in the gas phase: Synthesis, authentication, and thermochemistry, J. Am. Chem. Soc., 118, 11865-11871 (1996).

${ }^{67}$ P. G. Wenthold, R. R. Squires, and W. C. Lineberger, Ultraviolet photoelectron spectroscopy of the o-, m-, and p-benzyne negative ions. Electron affinities and singlet-triplet splittings for o-, m-, and p-benzyne, J. Am. Chem. Soc., 120, 5279-5290 (1998).

${ }^{68}$ M. J. Molina and F. S. Rowland, Stratospheric sink for chlorofluoromethanes: chlorine atom-catalysed destruction of ozone, Nature, $\mathbf{2 4 9}$ 810-812 (1974)

${ }^{69} \mathrm{Z}$. W. Qu, H. Zhu, and R. Schinke, Infrared spectrum of cyclic ozone: A theoretical investigation, J. Chem. Phys., 123(20), 204324 (2005).

${ }^{70}$ A. D. Chien, A. A. Holmes, M. Otten, C. J. Umrigar, S. Sharma, and P. M. Zimmerman, Excited states of methylene, polyenes, and ozone from heatbath configuration interaction, J. Phys. Chem. A, 122, 2714-2722 (2018).

${ }^{71}$ D. Theis, I. Joseph, T. L. Windus, and K. Ruedenberg, The transition from the open minimum to the ring minimum on the ground state and on the lowest excited state of like symmetry in ozone: A configuration interaction study, J. Chem. Phys., 144, 104304 (2019).

${ }^{72} \mathrm{~W}$. R. Harshbarger, Identification of the $\tilde{\mathrm{C}}$ state of ammonia by electron impact spectroscopy, J. Chem. Phys., 54, 2504-2509 (1971).

${ }^{73} \mathrm{M}$. B. Arfa and M. Tronc, Lowest energy triplet states of group Vb hydrides: $\mathrm{NH}_{3}\left(\mathrm{ND}_{3}\right)$ and $\mathrm{PH}_{3}$, Chem. Phys., 155, 143-148 (1991).

${ }^{74}$ K. Ralphs, G. Serna, L. R. Hargreaves, M. A. Khakoo, C. Winstead, and V. McKoy, Excitation of the six lowest electronic transitions in water by 9-20 eV electrons, J. Phys. B, 46, 125201 (2013).

${ }^{75} \mathrm{~A}$. Köhn and A. Tajti, Can coupled-cluster theory treat conical intersections?, J. Chem. Phys., 127, 044105 (2007).

${ }^{76} \mathrm{C}$. Hättig, Structure optimizations for excited states with correlated secondorder methods: $\mathrm{CC} 2$ and $\mathrm{ADC}(2), \mathrm{In} \mathrm{H}$. J. A.. Jensen, editor, Response Theory and Molecular Properties (A Tribute to Jan Linderberg and Poul Jorgensen), volume 50 of Advances in Quantum Chemistry, pages 37 - 60, Academic Press, (2005)

${ }^{77}$ E.F. Kjønstad, R.H. Myhre, T.J. Martínez, and H. Koch, Crossing conditions in coupled cluster theory, J. Chem. Phys., 147, 164105 (2017).

${ }^{78}$ E.F. Kjønstad and H. Koch, Resolving the notorious case of conical intersections for coupled cluster dynamics, J. Phys. Chem. Lett., 8, 4801-4807 (2017). 
${ }^{79}$ S. Faraji, S. Matsika, and A. I. Krylov, Calculations of non-adiabatic couplings within equation-of-motion coupled-cluster framework: Theory, implementation, and validation against multi-reference methods, J. Chem.
Phys., 148, 044103 (2018).

${ }^{80}$ E. F. Kjønstad and H. Koch, An orbital invariant similarity constrained coupled cluster model, J. Chem. Theory Comput., 15(10), 5386-5397 (2019). 